\title{
Influence of biofertilizers on protein, moisture and ash content in relation to swelling property, water absorption capacity, mineral elements, total phenolic level of Stevia (Stevia rebaudiana Bert.) plant grown under acidic soil zone of South India
}

\author{
Kuntal Das ${ }^{1, *}$, Raman Dang ${ }^{2}$ \\ 1 Departemnt of Pharmacognosy and Phytochemistry, Krupanidhi College of Pharmacy, \\ Carmelaram Post, Varthur Hobli, Bangalore - 35, India \\ Phon Mob: +919632542846 \\ ${ }^{2}$ Krupanidhi College of Pharmacy, Carmelaram Post, Varthur Hobli, Bangalore - 35, India \\ *E-mail address: drkkdsd@gmail.com
}

\begin{abstract}
The field experiment on Stevia rebaudiana (SR), (Family: Asteraceae) was carried out in acid soil zone of Shimoga (Karnataka) in the year 2009 and 2010 to investigate the influence of biofertilizers on protein, moisture and ash content in the dried Stevia leaf and their correlation with swelling property, water absorption capacity and mineral element contents. Results revealed that second harvested sample (August) in first year (2009) was better for all aspects than other harvested samples and the results showed significant increase in protein content $(16.22 \%)$, swelling index (SI) $(5.10 \% \mathrm{w} / \mathrm{w})$, water absorption capacity $(\mathrm{WAC})(4.91 \mathrm{ml} / \mathrm{g})$ with the treatment $\mathrm{T}_{8}$ where three bio fertilizers were applied togetherly with simultaneous moisture content varied from 6.61 to $7.18 \%$. The significant higher correlations were observed (significant at $1 \%)$ between protein content with SI ( $\mathrm{r}=$ $0.99)$, WAC $(r=0.99)$ and SI with WAC $(r=0.98)$ in 2009 harvested samples. The ash content was higher in third harvested sample (13.54\%) in the year 2009 with simultaneous increased amount of $\mathrm{Fe}$ $(6.19 \mathrm{mg} / \mathrm{kg}), \mathrm{Mn}(1.24 \mathrm{mg} / \mathrm{kg}), \mathrm{Cu}(0.72 \mathrm{mg} / \mathrm{kg})$ and $\mathrm{Zn}(1.22 \mathrm{mg} / \mathrm{kg})$ contents due to the residual effect of bio fertilizers. Nitrogen $(0.74 \mathrm{mg} / \mathrm{kg}), P(0.40 \mathrm{mg} / \mathrm{kg})$ and $\mathrm{K}(0.68 \mathrm{mg} / \mathrm{kg})$ contents were higher during second harvested samples and thereafter decreased. Total phenolic content was also recorded highest $(64.52 \mathrm{mg} / \mathrm{g})$ with three bio fertilizers applied togetherly during year 2009 compared to the year $2010(50.12 \mathrm{mg} / \mathrm{g})$. The results suggest that the application of bio-fertilizers either single or more numbers in a balanced way may be beneficial in improving protein, phenol and mineral contents of stevia plant.
\end{abstract}

Keywords: Atomic Absorption Spectrophotometer; Biofertilizers; Stevia rebaudiana; physiochemical properties; elements; phenols

\section{INTRODUCTION}

S. rebaudiana Bertoni (Family: Asteraceae) is an ancient South American perennial plant (native to Paraguay and Brazil) with great potential as an agricultural crop for the production of a high-potency natural sweetener (Soejarto, 2002). It is the source of a number 
of sweet ent-kaurene diterpenoid glycosides namely Stevioside, rebaudioside A.rebaudioside $\mathrm{B}$, rebaudioside $\mathrm{C}$, rebaudioside $\mathrm{E}$, and dulcoside A. (Prakash et al., 2008). Among that stevioside is mainly present (about 4-20\%) in dry-leaf matter (Ghanta et al., 2007) of Stevia leaf. Stevioside has been used as intensive more energetic sweeteners (have non caloric) in many countries of South America and Asia. The potential medicinal uses of Stevia are hypoglycemic, cardiovascular, antimicrobial, digestive tonic, dental and skin care (Mowrey, 1992). It is grown commercially in many parts of Brazil, Paraguay, Uruguay, Central America, Israel, Thailand and China. It was reported that the amounts of active components are dependent on total biomass yield, climatic feature, method of agro-techniques, water management and also fertilizer applications (Fronza \& Folegatti, 2003; Megeji et al., 2005). Even herbal drug industry requires large quantities of biomass for extraction of the sweetening compounds and hence need to enhance its biomass production through improved cultural techniques and application of manures and fertilizers including biofertilizers. Considering the importance, the stevia plant has domesticated in India in late $20^{\text {th }}$ century and commercially cultivated in few states of India especially in Gujarat, Karnataka, Tamil Nadu, West Bengal, and Uttar Pradesh. Recently, Vesicular Arbuscular Mycorrhiza (VAM), Phosphate Solubilizing Bacteria (PSB) and Azospirillum (AZO) are the most widespread biofertilizers significantly contributing macronutrients $(\mathrm{N}, \mathrm{P}$ and $\mathrm{K})$ and micronutrients (Fe, $\mathrm{Mn}$, $\mathrm{Cu}$ and $\mathrm{Zn}$ ) to plants (Smith and Read, 1997) and increased biomass with directly related to the better growth of the plant for commercial cultivation.

Phosphorus-solubilizers are biofertilizers which solubilize the adsorbed or fixed phosphorus in soil and make it available to plants. Azospirillum, heterotrophic nitrogen fixing organism, has been reported to be beneficial and economical on several crops. Bio fertilizers improved the growth, yield and protein content as well as the productivity of the crop. While, VAM fungi are the best known for their ability to improve plant growth in low phosphate soils by exploiting large areas of soil and actively transporting the phosphate back to the plants. It also increases the conditions of soil, uptake of $\mathrm{Zn}$ and $\mathrm{P}$, improve nitrogen fixation, $\mathrm{pH}$ tolerance, increases the uptake of immobilized nutrients and finally improves the crop growth. Along with the biofertilizers, farm yard manures (FYM) are also mixed with the soil to control the deficiency of macro and micronutrient contents in soil and increases the water holding capacity of the soil to stimulate the activity of beneficial microorganisms making unavailable nutrients available to the plants. Bio fertilizer application enhances the protein content and it is evident that proteins increase water holding capacity, when their swelling ability is enhanced. Water holding capacity is an important function of protein in viscous foods such as soups, gravies, doughs, and baked products. Hence, there is a need to develop a suitable low cost technology for better agricultural crop production. Apart from this, bio fertilizer also increases the phenolic content in the crop which are important for normal growth, development and defense against infection and injury. The presence of phenolic compounds in injured plants imparts the oxidative stability, microbial safety and important to human health because of their antioxidant potency (Hollman et al., 1996).

Keeping these in view, the present field investigation was undertaken to evaluate potentiality of Stevia plant about its functional, health promoting properties and raw materials for industry under improved agronomic management practices coupled with bio fertilizer application. 


\section{MATERIALS AND METHODS}

\section{1. Description of experimental sites and its cropping history}

This study was conducted at Ripponpet farm, Shimoga (latitudes $13^{\circ} 27^{\prime}$ and $14^{\circ} 39^{\prime} \mathrm{N}$ and between the longitudes $74^{\circ} 38^{\prime}$ and $76^{\circ} 04^{\prime} \mathrm{E}$ at a mean altitude of 640 metres above sea level) during 2009 and 2010. The soil at the Ripponpet farm has a sandy loam texture, with relatively high fertility, $\mathrm{pH} 6.10$. The area usually receives mean annual rainfall of $1815 \mathrm{~mm}$ and daily temperature range of 26 to $30^{\circ} \mathrm{C}$, but in summer time it reaches to $40{ }^{\circ} \mathrm{C}$.

\section{2. Details of experimental design and field layout}

Cuttings of Stevia plants, collected from Shimoga district, Karnataka, India, were used as a test plant for the present study. The field experiments were conducted in the month of February 2009 and data were collected for two years. Experimental fields were divided into beds according to treatments. The results of pre-planting surface $(0-15 \mathrm{~cm})$ soil analysis from the experimental plot was analyzed as $\mathrm{pH} 6.10$, organic carbon $0.54 \%$, cation exchange capacity (CEC) $9.89 \mathrm{cmol}\left(\mathrm{p}^{+}\right) / \mathrm{kg}$, available $\mathrm{N}, \mathrm{P}$ and $\mathrm{K}$ were $55.89 \mathrm{mg} / \mathrm{kg}, 6.8 \mathrm{mg} / \mathrm{kg}$ and $86.4 \mathrm{mg} / \mathrm{kg}$. DTPA extractable $\mathrm{Zn}, \mathrm{Cu}, \mathrm{Fe}$ and $\mathrm{Mn}$ were $0.54 \mathrm{mg} / \mathrm{kg}, 0.15 \mathrm{mg} / \mathrm{kg}, 5.2 \mathrm{mg} / \mathrm{kg}$ and $3.4 \mathrm{mg} / \mathrm{kg}$. Based on that results, and as per advise of agronomist, the following treatments were used: $T_{1}-$ Control, only FYM (farmyard manure) at $10 \mathrm{~kg}$, without application of biofertilizers; $\mathrm{T}_{2}$ - Soil application of FYM at $10 \mathrm{~kg}$ mixed with $250 \mathrm{~g}$ of PSB (phosphorus solubilizing bacteria, Bacillus megatheriam); $\mathrm{T}_{3}-$ Soil application of FYM at 10 $\mathrm{kg}$ mixed with $250 \mathrm{~g}$ of AZO (Azospirillum); $\mathrm{T}_{4}-$ Soil application of FYM at $10 \mathrm{~kg}$ mixed with $500 \mathrm{~g}$ of VAM (Vesicular arbuscular mycorrhiza); $\mathrm{T}_{5}$ - Soil application of FYM at 10 $\mathrm{kg}$ mixed with $250 \mathrm{~g}$ of VAM and $250 \mathrm{~g}$ of AZO; $\mathrm{T}_{6}-$ Soil application of FYM at $10 \mathrm{~kg}$ mixed with $250 \mathrm{~g}$ of PSB and $500 \mathrm{~g}$ of AZO; $\mathrm{T}_{7}-$ Soil application of FYM at $10 \mathrm{~kg}$ mixed with $250 \mathrm{~g}$ of PSB and $500 \mathrm{~g}$ of VAM; $\mathrm{T}_{8}-$ Soil application of FYM at $10 \mathrm{~kg}$ mixed with 250 $\mathrm{g}$ of PSB, $250 \mathrm{~g}$ of $\mathrm{AZO}$ and $500 \mathrm{~g}$ of VAM.

Each treatment was replicated thrice in a completely randomized design (CRD). Altogether, there were 24 ( 8 X 3) beds, with each bed size of 1 M X 1.25 M. Cutting of Stevia plants were planted in all the beds. Each bed contains 864 (36 X 24) plants with 6 rows and 6 columns (6X6). The moisture content was maintained at $60-70 \%$ of water holding capacity (WHC) by irrigating as per requirements.

\section{3. Plant sampling}

Fresh leaf samples at an interval of three months were collected (May, August and November) for continuous two years, dried and was analyzed separately for various physicochemical parameters. Year wise pooled biomass was collected separately (as per treatment wise) and analyzed for SI, WAC, element contents and total phenol content.

\section{4. Preparation of Stevia leaves}

The Stevia plants that reached (the maximum growth stage every after 3 months intervals) were harvested by cutting the plant at $5 \mathrm{~cm}$ from the cultivated plots (flowering was avoided by cutting the tops every 15 days alternate). The brown and yellow leaves were removed from the plants then, washed in clean water and spread on trays covered with cheese-cloth to remove the excess of water. The plants were dried in an oven temperature at 35-40 ${ }^{\circ} \mathrm{C}$ for $4-8 \mathrm{~h}$. Dried leaf samples were coarsely powdered, weighed and stored in 
moisture free sealed plastic cover in a dust proof cupboard for the further investigations. The yields of the pooled dried leaf biomass in the year 2009 and 2010 were presented in Table 7.

\section{5. Preparation of extract}

An aqueous extraction was performed for dried powdered Stevia leaves. $100 \mathrm{~g}$ dried leaves were soaked with $2.5 \mathrm{~g}$ of $\mathrm{CaCO}_{3}$ at $\mathrm{pH} 10$ for 8 hours and removed the impurities. All samples were refluxed with water for $4 \mathrm{hrs}$ after standardized the method. Finally crude extract was collected after filtration followed by rotary flash evaporation at $45{ }^{\circ} \mathrm{C}$ and were stored in well closed glass bottles in refrigeration condition at $4{ }^{\circ} \mathrm{C}$. The crude extracts were used for further investigation.

\section{6. Physicochemical assessment}

Chemical analysis to determine proximate composition of sample was carried out using standard procedure. Moisture content was determined by air drying at $105{ }^{\circ} \mathrm{C}$ until a constant weight was attained (AOAC, 1990), proteins content was determined from the nitrogen content by Kjeldahl method (Regal's 234, supplied by Popular Science Apparatus Workshops Pvt Ltd, India) (AOAC, 978.04) using factor 6.25 and calculated as $\mathrm{N} x 6.25$ (AOAC, 1995), swelling property was determined by measuring the volume difference between before and after swelling by soaking in water for overnight (Mukherjee, 2007), water absorption capacity was determined by method described by Rosario and Hores (1981), ash contents by incineration method at a temperature $450{ }^{\circ} \mathrm{C}$, mineral elements composition estimated using the atomic absorption spectrophotometer (AAS) after acid digestion of the samples. All the results were expressed as \% on dry weight basis.

\section{7. Elemental analysis}

Essential and non essential element contents, i.e. nitrogen $(\mathrm{N})$, copper $(\mathrm{Cu})$, manganese $(\mathrm{Mn})$, iron $(\mathrm{Fe})$ and zinc $(\mathrm{Zn})$ were determined from aliquots of the ash solutions (after digestion with acid mixtures) according to the method of AOAC (2000) using Kjeldahl method (for N) and AAS. Further Determination of phosphorus (P) content in plant was done by using a spectrophotometer (ECIL, made in India) after development of yellow color with vanado-molybdo phosphoric acid and then the intensity of color was determined at $420 \mathrm{~nm}$ (blue filter) and potassium (K) content in plant samples was determined by using Flame Photometer (Elico, made in India) after plant samples $(0.5 \mathrm{~g})$ were digested with a di-acid mixture $\left(\mathrm{H}_{2} \mathrm{SO}_{4}: \mathrm{HClO}_{4}: 6: 4\right)$.

\section{8. Atomic absorption spectrophotometer}

Content of the mineral elements in the dried leaf sample of cultivated Stevia were measured with double-beam atomic absorption spectrophotometer (Model-Aanalyst 100, supplied by Perkin Elmer, USA). The main source used for AA was the hollow cathode lamp (HCL). The dual option burner system was used with flow spoiler in a spray chamber. The common oxidant/fuel combination used in AA was air-acetylene. The best wavelengths were selected for the analysis based on the concentration ranges of sample. The slit width $(0.2 \mathrm{~nm})$ was the optimum for the elements which are determined. The following conditions were selected for said metals analysis: 


\begin{tabular}{|c|c|c|c|}
\hline Elements & $\begin{array}{c}\text { Wavelength } \\
(\mathrm{nm})\end{array}$ & Ash Temperature $\left({ }^{\circ} \mathrm{C}\right)$ & $\begin{array}{c}\text { Atomization } \\
\text { Temperature }\left({ }^{\circ} \mathrm{C}\right)\end{array}$ \\
\hline $\mathrm{Mn}$ & 279.5 & 800 & 2400 \\
\hline
\end{tabular}

Flame Atomic Absorption System

\begin{tabular}{|c|c|l|}
\hline Elements & Wave length & Gas mixture \\
\hline $\mathrm{Cu}$ & 327.4 & Air-acetylene \\
\hline $\mathrm{Fe}$ & 372.0 & Air-acetylene \\
\hline $\mathrm{Zn}$ & 213.9 & Air-acetylene \\
\hline
\end{tabular}

The concentration of the above said elements was determined by using the standard conditions. The method was based upon the linear relation between the absorbance (AU) and concentration of the determined element. Samples were made in triplicate. The obtained results are expressed in $\mathrm{mg} / \mathrm{kg}$ on a dry mass weight in sample.

\subsection{Determination of total phenolic content}

Phenolic content in the leaf extract (aqueous extract) of SR were determined with Folin-Ciocalteau reagent according to the method described by Slinkard and Singleton (1977). Gallic acid was used as a standard. About $1.0 \mathrm{ml}$ of extract solution containing $1.0 \mathrm{~g}$ extract in a volumetric flask was diluted with $46 \mathrm{ml}$ of distilled water. About $1.0 \mathrm{ml}$ of FolinCiocalteau reagent was added and mixed. After few minutes $3.0 \mathrm{ml}$ of $2 \%$ sodium carbonate was added and then the mixture was allowed to stand for $3 \mathrm{~h}$ with occasional shaking. The absorbance of the blue color that developed was measured at $760 \mathrm{~nm}$. The concentration of total phenols was expressed as $\mathrm{mg} / \mathrm{g}$ of dry extract (Kim et al., 2003) that was determined as $\mathrm{mg}$ of Gallic acid equivalent per $\mathrm{g}$ using the equation, taken form prepared standard Gallic acid calibration curve $\mathrm{y}=15.27 \mathrm{x}+12.44, \mathrm{R}^{2}=0.997$.

\section{10. Statistical Analysis}

The results of the field experiment were expressed as mean \pm standard error of mean (SEM) of three replicates. The data were subjected to one way analysis of variance (ANOVA) and differences between samples were determined by using Graph Prism Pad 5 software. The values showing $\mathrm{p}<0.05$ were regarded as significant. All results further analyzed by post test (Dunnett comparison test) for the significant results.

\section{RESULTS}

\section{1. Physicochemical assessment}

The First harvested sample in May, 2009, where crude protein content was higher with the treatment eight $(16.22 \%)$ followed by treatment seven $(16.12 \%)$ where combinations of three and two biofertilizers were applied respectively. All the values were even higher than 
control $(12.22 \%)$ treatment. Ash content, SI and WAC were also followed the same trend as like protein, being higher in $\mathrm{T}_{8}$ treatment with ash, SI, and WAC contents of $9.13 \%, 5.10 \%$ $\mathrm{w} / \mathrm{w}$ and WAC $4.91 \mathrm{mg} / \mathrm{ml}$ respectively. Moisture content was varied with the treatments being higher with treatment four $(7.18 \%)$ where VAM biofertilizers was applied followed by $7.15 \%$ with the $T_{5}$ and $T_{7}$ respectively. Second harvested sample in 2009 were also showed the same trend as first harvested sample with respect to applied biofertilizers.

Fig. 1. Comparative effect biofertilizers on protein content during in two different years.

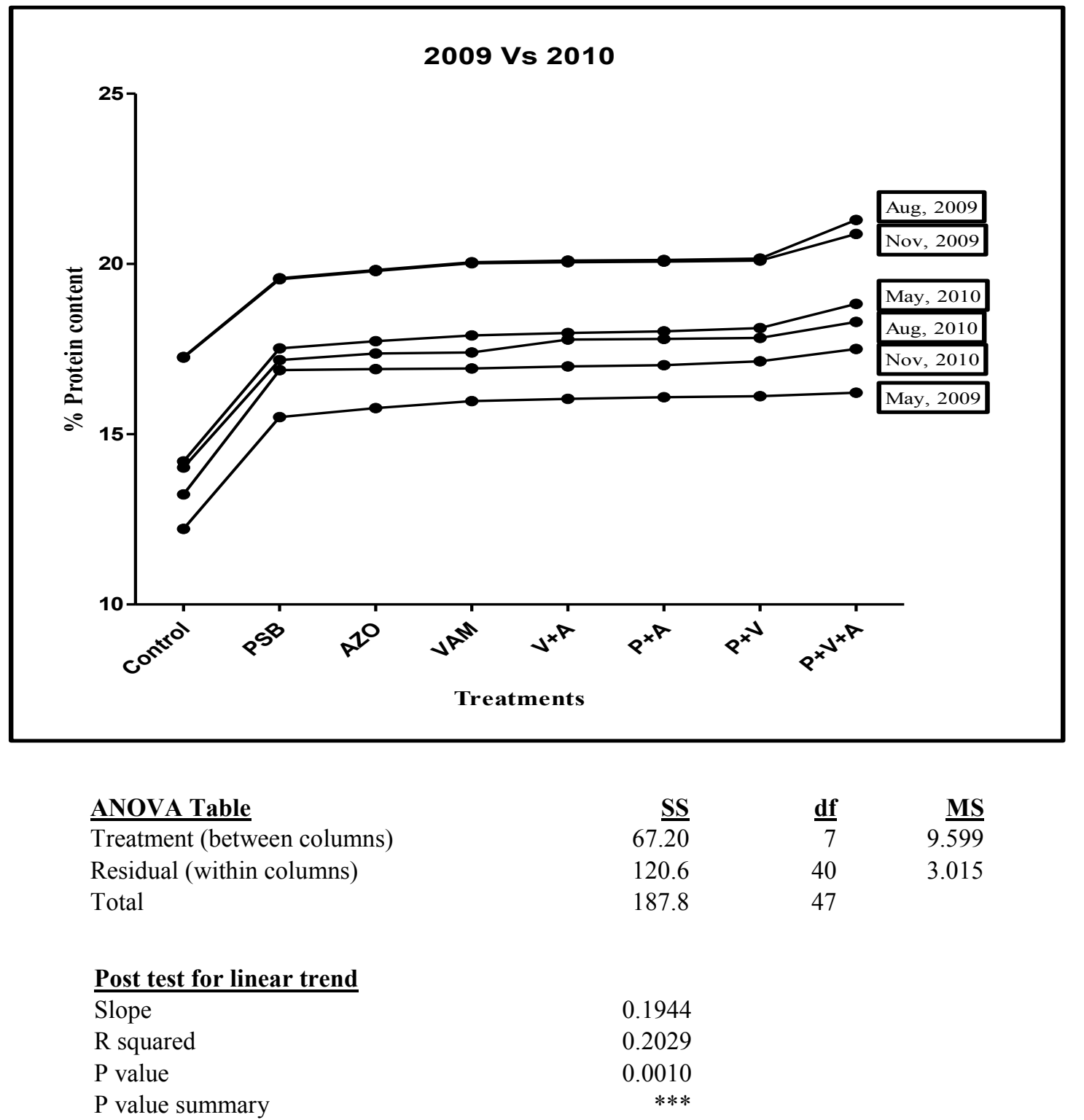

The highest protein content $(21.29 \%)$, SI $(6.42 \%)$, WAC $(5.12 \mathrm{ml} / \mathrm{g})$, ash content (13.43\%) were reported with $\mathrm{T}_{8}$ from second harvested sample in August, 2009 whereas same has been reported with $20.88 \%, 6.22 \%, 4.99 \mathrm{ml} / \mathrm{g}$ and $13.54 \%$ in third harvested sample collected in November, 2009 which were higher than control sample but moisture contents were varied with the applied biofertilizers in both the dried leaf samples in the range 
of 6.91 to $7.00 \%$ from both the harvested crops. In case of harvested sample in 2010 , there were significant changes observed for protein content, ash value, SI and WAC whereas no significant changes observed with moisture content. The fourth harvested sample in May 2010, resulted protein content (18.83\%), ash value (13.43\%), SI (6.18 \%) and WAC (4.97 $\mathrm{ml} / \mathrm{g}$ ) were higher with same treatments. Fifth and sixth harvested samples in 2010 were also followed the same trend, being high protein content 18.30 and $17.50 \%$; ash content 12.99 and $10.29 \%$; SI 6.11 and $6.04 \%$; and WAC 4.94 and $4.86 \mathrm{ml} / \mathrm{g}$ in fifth (August) and sixth (November) harvested samples respectively with treatment eight in the year 2010 but the results were lesser than that of former results. Effect of biofertilizers on protein content during two different years was depicted in Figure 1 and clearly showed higher protein content during year 2009 with second harvested samples. SI and WAC of dried Stevia leaves were also reported higher than other harvested samples (Figure $2 \& 3$ ).

Fig. 2. Determination of swelling index from all the harvested Stevia leaf in 2009 and 2010.

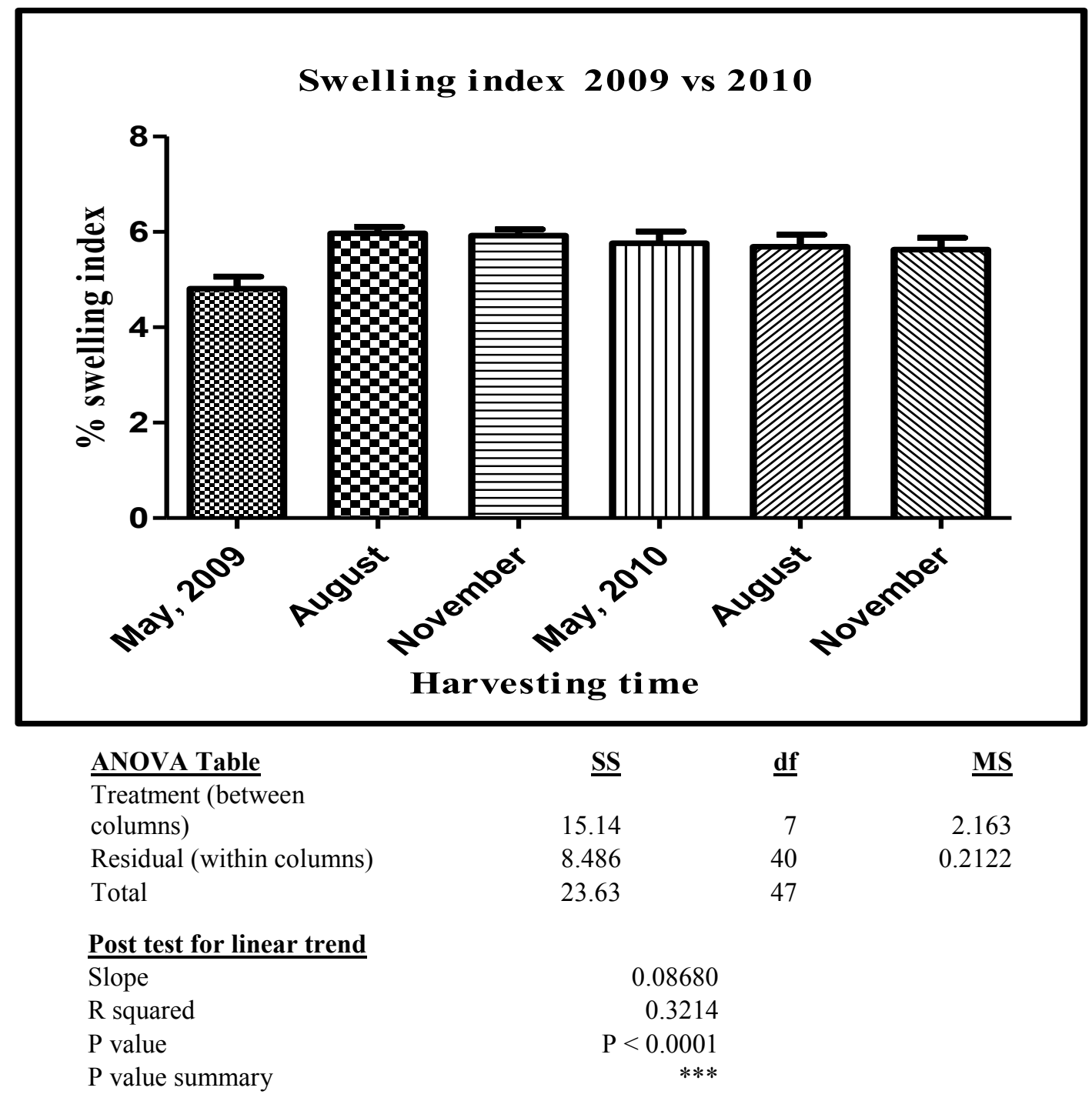

Fig. 3. Determination of water absorption capacity from all the harvested Stevia leaf in 2009 and 2010. 


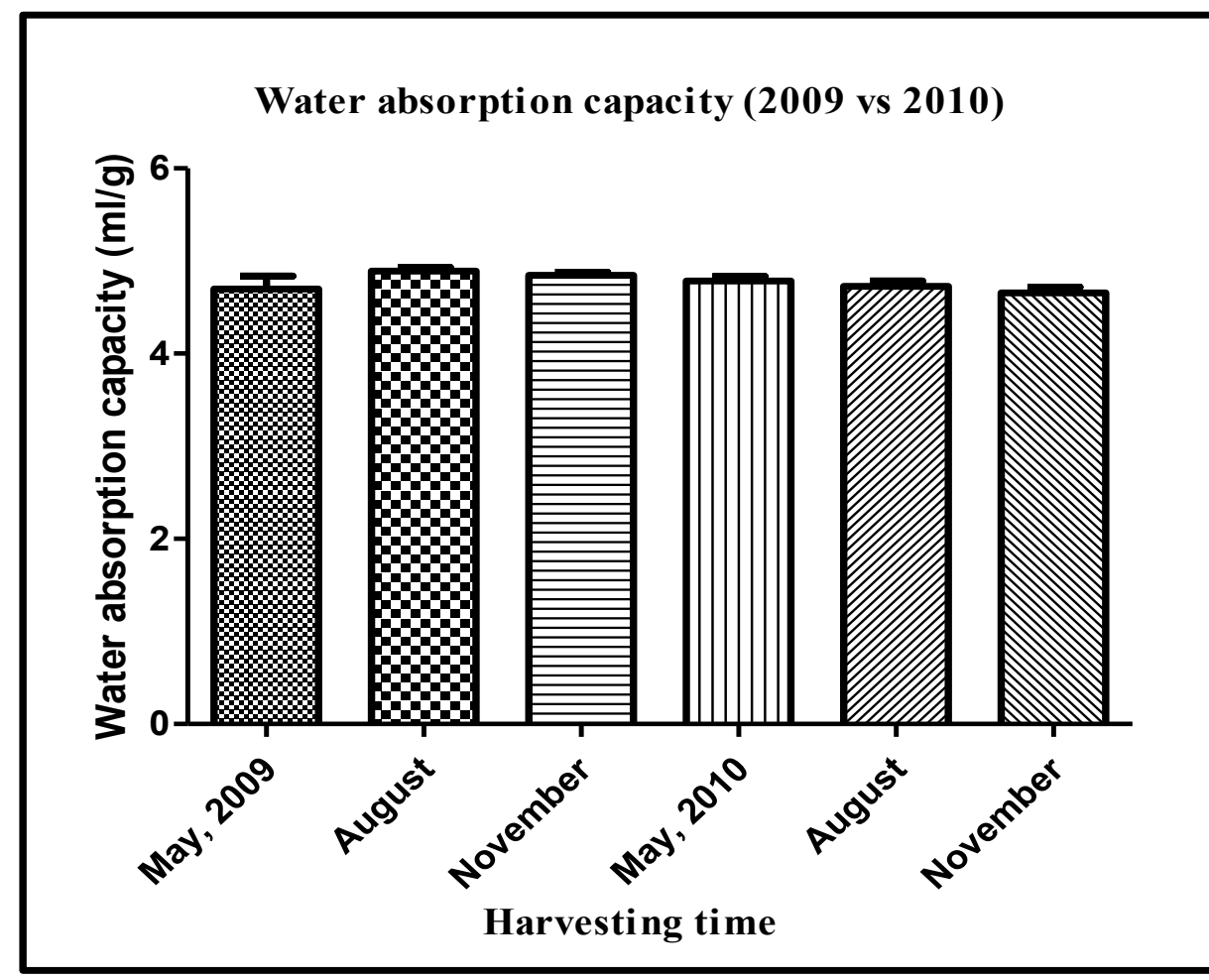

\section{ANOVA Table}

Treatment (between columns)

Residual (within columns)

Total

$\begin{array}{rrr}\underline{\mathbf{S S}} & \underline{\mathbf{d f}} & \underline{\mathbf{M S}} \\ 1.183 & 7 & 0.1690 \\ 0.9197 & 40 & 0.02299 \\ 2.103 & 47 & \end{array}$

$$
\begin{array}{r}
0.03029 \\
0.4398 \\
\mathrm{P}<0.0001
\end{array}
$$

$\mathrm{P}$ value summary

Table 1. Correlation matrix between mean \% protein content, swelling index and water absorption capacity in both the years.

\begin{tabular}{|c|c|c|c|c|c|c|}
\hline \multirow{2}{*}{ Parameters } & \multicolumn{3}{|c|}{2009} & \multicolumn{3}{c|}{2010} \\
\cline { 2 - 7 } & Protein & $\begin{array}{c}\text { Swelling } \\
\text { Index }\end{array}$ & $\begin{array}{c}\text { Water } \\
\text { absorption } \\
\text { capacity }\end{array}$ & Protein & $\begin{array}{c}\text { Swelling } \\
\text { Index }\end{array}$ & $\begin{array}{c}\text { Water } \\
\text { absorption } \\
\text { capacity }\end{array}$ \\
\hline Protein & 1.00 & & & 1.00 & & \\
\hline $\begin{array}{c}\text { Swelling } \\
\text { Index }\end{array}$ & $0.99^{* *}$ & 1.00 & & $0.99^{* *}$ & 1.00 & \\
\hline $\begin{array}{c}\text { Water } \\
\text { absorption } \\
\text { capacity }\end{array}$ & $0.99^{* *}$ & $0.98^{* *}$ & 1.00 & $0.93^{*}$ & $0.91^{*}$ & 1.00 \\
\hline
\end{tabular}

\footnotetext{
${ }^{* *}$ Significant at $1 \%$; "Significant at $5 \%$
} 
Correlation matrices among protein content, SI and WAC were showed in Table 1, and showed highly significant positive correlation (Significant at $1 \%$ ) in year 2009 than in year 2010 .

\section{2. Elemental analysis}

Table 2 revealed the various microelements contents in the dried Stevia plant in year 2009.

Table 2. Elemental analysis (Micro) in dried Stevia leaf in 2009 (Mean \pm SEM, $\mathrm{n}=3$, from three replicated plots).

\begin{tabular}{|c|c|c|c|c|c|c|c|c|c|c|c|c|}
\hline \multirow[t]{2}{*}{ Ts } & \multicolumn{4}{|c|}{$\begin{array}{l}\text { After first harvest (May) } \\
\text { (mg kg-1, dry wt basis) }\end{array}$} & \multicolumn{4}{|c|}{$\begin{array}{l}\text { After second harvest (August) } \\
\qquad\left(\mathrm{mg} \mathrm{kg}^{-1}, \text { dry wt basis }\right)\end{array}$} & \multicolumn{4}{|c|}{$\begin{array}{l}\text { After third harvest (November) } \\
\qquad\left(\mathrm{mg} \mathrm{kg}^{-1}, \text { dry wt basis) }\right.\end{array}$} \\
\hline & $\mathrm{Fe}$ & $\mathrm{Mn}$ & $\mathrm{Cu}$ & $\mathrm{Zn}$ & $\mathrm{Fe}$ & $\mathrm{Mn}$ & $\mathrm{Cu}$ & $\mathrm{Zn}$ & $\mathrm{Fe}$ & Mn & $\mathrm{Cu}$ & $\mathrm{Zn}$ \\
\hline \multirow{2}{*}{$\mathrm{T}_{1}$} & $3.09 \pm$ & $0.54 \pm$ & $0.46 \pm$ & $0.58 \pm$ & $3.13 \pm$ & $0.55 \pm$ & $0.45 \pm$ & $0.59 \pm$ & $3.09 \pm$ & $0.59 \pm$ & $0.43 \pm$ & $0.58 \pm$ \\
\hline & 0.017 & 0.008 & 0.008 & 0.003 & 0.006 & 0.005 & 0.006 & 0.003 & 0.012 & 0.003 & 0.003 & 0.005 \\
\hline \multirow{2}{*}{$\mathrm{T}_{2}$} & $3.08 \pm$ & $0.58 \pm$ & $0.47 \pm$ & $0.61 \pm$ & $4.19 \pm$ & $0.67 \pm$ & $0.50 \pm$ & $0.65 \pm$ & $4.21 \pm$ & $0.70 \pm$ & $0.54 \pm$ & $0.72 \pm$ \\
\hline & $0.011^{\mathrm{ns}}$ & $0.005^{*}$ & $0.003^{\text {ns }}$ & $0.006^{*}$ & $0.008^{* * *}$ & $0.008^{* * *}$ & $0.003^{* * *}$ & $0.006^{* * *}$ & $0.008^{* * *}$ & $0.005^{* * *}$ & $0.008^{* * *}$ & $0.033^{* * *}$ \\
\hline \multirow{2}{*}{$\mathrm{T}_{3}$} & $3.17 \pm$ & $0.62 \pm$ & $0.50 \pm$ & $0.65 \pm$ & $4.27 \pm$ & $0.74 \pm$ & $0.50 \pm$ & $0.69 \pm$ & $4.30 \pm$ & $0.77 \pm$ & $0.59 \pm$ & $0.73 \pm$ \\
\hline & $0.008^{\text {ns }}$ & $0.008^{* * *}$ & $0.008^{* *}$ & $0.010^{* * *}$ & $0.008^{* * *}$ & $0.012^{* * *}$ & $0.005^{* * *}$ & $0.005^{* * *}$ & $0.008^{* * *}$ & $0.006^{* * *}$ & $0.005^{* * *}$ & $0.003^{* * *}$ \\
\hline \multirow{2}{*}{$\mathrm{T}_{4}$} & $3.13 \pm$ & $0.57 \pm$ & $0.51 \pm$ & $0.64 \pm$ & $4.20 \pm$ & $0.71 \pm$ & $0.51 \pm$ & $0.67 \pm$ & $4.25 \pm$ & $0.74 \pm$ & $0.55 \pm$ & $0.73 \pm$ \\
\hline & $0.014^{\mathrm{ns}}$ & $0.008^{\mathrm{ns}}$ & $0.003^{* * *}$ & $0.005^{\mathrm{ns}}$ & $0.012^{* * *}$ & $0.008^{* * *}$ & $0.006^{* * *}$ & $0.005^{* * *}$ & $0.006^{* * * *}$ & $0.008^{* * *}$ & $0.003^{* * *}$ & $0.005^{* * *}$ \\
\hline \multirow{2}{*}{$\mathrm{T}_{5}$} & $3.22 \pm$ & $0.68 \pm$ & $0.57 \pm$ & $0.66 \pm$ & $4.85 \pm$ & $0.85 \pm$ & $0.59 \pm$ & $0.80 \pm$ & $4.87 \pm$ & $0.87 \pm$ & $0.61 \pm$ & $0.90 \pm$ \\
\hline & $0.043^{* *}$ & $0.008^{* * *}$ & $0.003^{* * *}$ & $0.008^{* * *}$ & $0.014^{* * *}$ & $0.010^{* * *}$ & $0.006^{* * *}$ & $0.008^{* * *}$ & $0.003^{* * *}$ & $0.006^{* * *}$ & $0.005^{* * *}$ & $0.014^{* * *}$ \\
\hline \multirow{2}{*}{$\mathrm{T}_{6}$} & $3.15 \pm$ & $0.69 \pm$ & $0.56 \pm$ & $0.63 \pm$ & $4.81 \pm$ & $0.86 \pm$ & $0.57 \pm$ & $0.88 \pm$ & $4.84 \pm$ & $0.90 \pm$ & $0.64 \pm$ & $0.97 \pm$ \\
\hline & $0.012^{\mathrm{ns}}$ & $0.003^{* * *}$ & $0.012^{* * *}$ & $0.005^{* * *}$ & $0.005^{* * *}$ & $0.003^{* * *}$ & $0.006^{* * *}$ & $0.008^{* * *}$ & $0.003^{* * *}$ & $0.005^{* * *}$ & $0.008^{* * *}$ & $0.011^{* * *}$ \\
\hline \multirow{2}{*}{$\mathrm{T}_{7}$} & $3.18 \pm$ & $0.66 \pm$ & $0.54 \pm$ & $0.65 \pm$ & $4.81 \pm$ & $0.89 \pm$ & $0.55 \pm$ & $0.87 \pm$ & $4.83 \pm$ & $0.91 \pm$ & $0.62 \pm$ & $1.00 \pm$ \\
\hline & $0.008^{*}$ & $0.006^{* * *}$ & $0.005^{* * *}$ & $0.008^{* * *}$ & $0.006^{* * *}$ & $0.003^{* * *}$ & $0.003^{* * *}$ & $0.008^{* * *}$ & $0.013^{* * *}$ & $0.006^{* * *}$ & $0.015^{* * *}$ & $0.005^{* * *}$ \\
\hline \multirow{2}{*}{$\mathrm{T}_{8}$} & $3.24+$ & $0.72 \pm$ & $0.60 \pm$ & $0.69 \pm$ & $6.13+$ & $1.21 \pm$ & $0.58 \pm$ & $1.15 \pm$ & $6.19+$ & $1.24 \pm$ & $0.72 \pm$ & $1.22 \pm$ \\
\hline & $0.011^{* * *}$ & $0.008^{* * *}$ & $0.003^{* * *}$ & $0.005^{* * *}$ & $0.006^{* * *}$ & $0.008^{* * *}$ & $0.008^{* * *}$ & $0.006^{* * *}$ & $0.010^{* * *}$ & $0.010^{* * *}$ & $0.012^{* * *}$ & $0.012^{* * *}$ \\
\hline
\end{tabular}

- $\quad$ Ts $=$ Treatments; $\mathrm{T}_{1}=$ Control $(\mathrm{FYM}), \mathrm{T}_{2}=\mathrm{FYM}+\mathrm{PSB}, \mathrm{T}_{3}=\mathrm{FYM}+\mathrm{AZO}, \mathrm{T}_{4}=\mathrm{FYM}+\mathrm{VAM}$,

$\mathrm{T}_{5}=\mathrm{FYM}+\mathrm{VAM}+\mathrm{AZO}$,

$\mathrm{T}_{6}=\mathrm{FYM}+\mathrm{PSB}+\mathrm{AZO}, \mathrm{T}_{7}=\mathrm{FYM}+\mathrm{PSB}+\mathrm{VAM}, \mathrm{T}_{8}=\mathrm{FYM}+\mathrm{PSB}+\mathrm{AZO}+\mathrm{VAM} ;$

- $\quad * \mathrm{P}<0.05, * * \mathrm{P}<0.01$ and $* * * \mathrm{P}<0.001 ; \mathrm{ns}=$ Non significant (compared with control treatment).

During third harvest of the samples in November showed higher accumulation of the elements than other treatments, being higher Fe content $6.19 \mathrm{mg} / \mathrm{kg}$, Mn content $1.24 \mathrm{mg} / \mathrm{kg}$, $\mathrm{Cu}$ content $0.72 \mathrm{mg} / \mathrm{kg}$ and $1.22 \mathrm{mg} / \mathrm{kg}$ with the treatment eight where combination of three biofertilizers were applied.

The values were even higher than control samples. The same trend were also followed in year 2010 where accumulation of Fe $(5.77 \mathrm{mg} / \mathrm{kg}), \mathrm{Mn}(1.18 \mathrm{mg} / \mathrm{kg}), \mathrm{Cu}(0.66 \mathrm{mg} / \mathrm{kg})$ and 
Zn $(1.16 \mathrm{mg} / \mathrm{kg})$ content were higher with the treatment eight in fifth harvested samples in May (Table 3) and further decreased drastically with rest of the harvested samples.

Table 3. Elemental analysis (Micro) in dried Stevia leaf in 2010 (Mean \pm SEM, $\mathrm{n}=3$, from three replicated plots).

\begin{tabular}{|c|c|c|c|c|c|c|c|c|c|c|c|c|}
\hline \multirow[t]{2}{*}{ Ts } & \multicolumn{4}{|c|}{$\begin{array}{l}\text { After first harvest (May) } \\
\text { (mg kg-1, dry wt basis) }\end{array}$} & \multicolumn{4}{|c|}{$\begin{array}{l}\text { After second harvest (August) } \\
\qquad\left(\mathrm{mg} \mathrm{kg}^{-1}, \text { dry wt basis) }\right.\end{array}$} & \multicolumn{4}{|c|}{$\begin{array}{l}\text { After third harvest (November) } \\
\qquad\left(\mathrm{mg} \mathrm{kg}^{-1} \text {, dry wt basis) }\right.\end{array}$} \\
\hline & $\mathrm{Fe}$ & $\mathrm{Mn}$ & $\mathrm{Cu}$ & $\mathrm{Zn}$ & $\mathrm{Fe}$ & $\mathrm{Mn}$ & $\mathrm{Cu}$ & $\mathrm{Zn}$ & $\mathrm{Fe}$ & $\mathrm{Mn}$ & $\mathrm{Cu}$ & $\mathrm{Zn}$ \\
\hline $\mathrm{T}_{1}$ & $\begin{array}{l}2.74 \pm \\
0.008\end{array}$ & $\begin{array}{l}0.49 \pm \\
0.011\end{array}$ & $\begin{array}{l}0.36 \pm \\
0.003\end{array}$ & $\begin{array}{l}0.52 \pm \\
0.033\end{array}$ & $\begin{array}{l}2.60 \pm \\
0.004\end{array}$ & $\begin{array}{l}0.40 \pm \\
0.004\end{array}$ & $\begin{array}{l}0.30 \pm \\
0.005\end{array}$ & $\begin{array}{l}0.46 \pm \\
0.004\end{array}$ & $\begin{array}{l}2.50 \pm \\
0.003\end{array}$ & $\begin{array}{l}0.30 \pm \\
0.008\end{array}$ & $\begin{array}{l}0.27 \pm \\
0.005\end{array}$ & $\begin{array}{l}0.40 \pm \\
0.006\end{array}$ \\
\hline $\mathrm{T}_{2}$ & $\begin{array}{c}4.02 \pm \\
0.012^{* * *}\end{array}$ & $\begin{array}{c}0.62 \pm \\
0.006^{* * *}\end{array}$ & $\begin{array}{c}0.50 \pm \\
0.005^{* * *}\end{array}$ & $\begin{array}{c}0.64 \pm \\
0.003^{* * *}\end{array}$ & $\begin{array}{c}4.00 \pm \\
0.010^{* * *}\end{array}$ & $\begin{array}{c}0.54 \pm \\
0.012^{* * *}\end{array}$ & $\begin{array}{c}0.42 \pm \\
0.010^{* * *}\end{array}$ & $\begin{array}{c}0.56 \pm \\
0.000^{* * *}\end{array}$ & $\begin{array}{c}3.10 \pm \\
0.011^{* * *}\end{array}$ & $\begin{array}{c}0.43 \pm \\
0.014^{* * *}\end{array}$ & $\begin{array}{c}0.36 \pm \\
0.003^{* * *}\end{array}$ & $\begin{array}{c}0.46 \pm \\
0.012^{* * *}\end{array}$ \\
\hline $\mathrm{T}_{3}$ & $\begin{array}{c}4.13 \pm \\
0.005^{* * *}\end{array}$ & $\begin{array}{c}0.72 \pm \\
0.004^{* * *}\end{array}$ & $\begin{array}{c}0.52 \pm \\
0.014^{* * *}\end{array}$ & $\begin{array}{c}0.65 \pm \\
0.004^{* * *}\end{array}$ & $\begin{array}{c}4.01 \pm \\
0.006^{* * *}\end{array}$ & $\begin{array}{c}0.58 \pm \\
0.008^{* * *}\end{array}$ & $\begin{array}{c}0.44 \pm \\
0.012^{* * *}\end{array}$ & $\begin{array}{c}0.57 \pm \\
0.003^{* * *}\end{array}$ & $\begin{array}{c}3.13 \pm \\
0.004^{* * *}\end{array}$ & $\begin{array}{c}0.45 \pm \\
0.012^{* * *}\end{array}$ & $\begin{array}{c}0.38 \pm \\
0.003^{* * *}\end{array}$ & $\begin{array}{c}0.48 \pm \\
0.012^{* * *}\end{array}$ \\
\hline $\mathrm{T}_{4}$ & $\begin{array}{c}4.19 \pm \\
0.005^{* * *}\end{array}$ & $\begin{array}{c}0.67 \pm \\
0.012^{* * *}\end{array}$ & $\begin{array}{c}0.49 \pm \\
0.003^{* * *}\end{array}$ & $\begin{array}{c}0.68 \pm \\
0.012^{* * *}\end{array}$ & $\begin{array}{c}4.04 \pm 0 . \\
012^{* * *}\end{array}$ & $\begin{array}{c}0.59 \pm \\
0.000^{* * *}\end{array}$ & $\begin{array}{c}0.47 \pm \\
0.012^{* * *}\end{array}$ & $\begin{array}{c}0.59 \pm \\
0.011^{* * *}\end{array}$ & $\begin{array}{c}3.18 \pm \\
0.008^{* * *}\end{array}$ & $\begin{array}{c}0.47 \pm \\
0.004^{* * *}\end{array}$ & $\begin{array}{c}0.42 \pm \\
0.011^{* * *}\end{array}$ & $\begin{array}{c}0.50 \pm \\
0.010^{* * *}\end{array}$ \\
\hline $\mathrm{T}_{5}$ & $\begin{array}{c}4.16 \pm \\
0.008^{* * *}\end{array}$ & $\begin{array}{c}0.76 \pm \\
0.005^{* * *}\end{array}$ & $\begin{array}{c}0.53 \pm \\
0.004^{* * *}\end{array}$ & $\begin{array}{c}0.84 \pm \\
0.010^{* * *}\end{array}$ & $\begin{array}{c}4.06 \pm 0 . \\
012^{* * *}\end{array}$ & $\begin{array}{c}0.63 \pm \\
0.012^{* * *}\end{array}$ & $\begin{array}{c}0.48 \pm \\
0.003^{* * *}\end{array}$ & $\begin{array}{c}0.68 \pm \\
0.006^{* * *}\end{array}$ & $\begin{array}{c}3.24 \pm \\
0.010^{* * *}\end{array}$ & $\begin{array}{c}0.49 \pm \\
0.010^{* * *}\end{array}$ & $\begin{array}{c}0.43 \pm \\
0.012^{* * *}\end{array}$ & $\begin{array}{c}0.54 \pm \\
0.008^{* * *}\end{array}$ \\
\hline $\mathrm{T}_{6}$ & $\begin{array}{c}4.57 \pm \\
0.015^{* * *}\end{array}$ & $\begin{array}{c}0.85 \pm \\
0.003^{* * *}\end{array}$ & $\begin{array}{c}0.57 \pm \\
0.012^{* * *}\end{array}$ & $\begin{array}{c}0.87 \pm 0.0 \\
06^{* * *}\end{array}$ & $\begin{array}{c}4.12 \pm 0 . \\
006^{* * *}\end{array}$ & $\begin{array}{c}0.72 \pm \\
0.004^{* * *}\end{array}$ & $\begin{array}{c}0.50 \pm \\
0.008^{* * *}\end{array}$ & $\begin{array}{c}0.69 \pm \\
0.004^{* * *}\end{array}$ & $\begin{array}{c}3.26 \pm \\
0.003^{* * *}\end{array}$ & $\begin{array}{c}0.53 \pm \\
0.005^{* * *}\end{array}$ & $\begin{array}{c}0.46 \pm \\
0.004^{* * *}\end{array}$ & $\begin{array}{c}0.57 \pm \\
0.006^{* * *}\end{array}$ \\
\hline $\mathrm{T}_{7}$ & $\begin{array}{c}4.71 \pm \\
0.006^{* * *}\end{array}$ & $\begin{array}{c}0.85 \pm \\
0.012^{* * *}\end{array}$ & $\begin{array}{c}0.59 \pm \\
0.006^{* * *}\end{array}$ & $\begin{array}{c}0.98 \pm \\
0.011^{* * *}\end{array}$ & $\begin{array}{c}4.52 \pm 0 \\
012^{* * *}\end{array}$ & $\begin{array}{c}0.74 \pm \\
0.006^{* * *}\end{array}$ & $\begin{array}{c}0.51 \pm \\
0.005^{* * *}\end{array}$ & $\begin{array}{c}0.79 \pm \\
0.010^{* * *}\end{array}$ & $\begin{array}{c}3.32 \pm \\
0.012^{* * *}\end{array}$ & $\begin{array}{c}0.57 \pm \\
0.004^{* * *}\end{array}$ & $\begin{array}{c}0.47 \pm \\
0.008^{* * *}\end{array}$ & $\begin{array}{c}0.67 \pm \\
0.011^{* * *}\end{array}$ \\
\hline $\mathrm{T}_{8}$ & $\begin{array}{c}5.77+ \\
0.015^{* * *}\end{array}$ & $\begin{array}{c}1.18 \pm \\
0.010^{* * *}\end{array}$ & $\begin{array}{c}0.66 \pm \\
0.010^{* * *}\end{array}$ & $\begin{array}{c}1.16 \pm \\
0.008^{* * *}\end{array}$ & $\begin{array}{c}5.17 \pm 0 . \\
008^{* * *}\end{array}$ & $\begin{array}{c}1.00 \pm \\
0.011^{* * *}\end{array}$ & $\begin{array}{c}0.56 \pm \\
0.012^{* * *}\end{array}$ & $\begin{array}{c}1.00 \pm \\
0.012^{* * *}\end{array}$ & $\begin{array}{c}4.28 \pm \\
0.003^{* * *}\end{array}$ & $\begin{array}{c}0.86 \pm \\
0.011^{* * *}\end{array}$ & $\begin{array}{c}0.49 \pm \\
0.010^{* * *}\end{array}$ & $\begin{array}{c}0.96 \pm \\
0.003^{* * *}\end{array}$ \\
\hline
\end{tabular}

- $\quad \mathrm{Ts}=$ Treatments; $\mathrm{T}_{1}=$ Control $(\mathrm{FYM}), \mathrm{T}_{2}=\mathrm{FYM}+\mathrm{PSB}, \mathrm{T}_{3}=\mathrm{FYM}+\mathrm{AZO}, \mathrm{T}_{4}=\mathrm{FYM}+\mathrm{VAM}$,

$\mathrm{T}_{5}=\mathrm{FYM}+\mathrm{VAM}+\mathrm{AZO}$

$\mathrm{T}_{6}=\mathrm{FYM}+\mathrm{PSB}+\mathrm{AZO}, \mathrm{T}_{7}=\mathrm{FYM}+\mathrm{PSB}+\mathrm{VAM}, \mathrm{T}_{8}=\mathrm{FYM}+\mathrm{PSB}+\mathrm{AZO}+\mathrm{VAM} ;$

- $\quad * \mathrm{P}<0.05, * * \mathrm{P}<0.01$ and $* * * \mathrm{P}<0.001 ; \mathrm{ns}=$ Non significant (compared with control treatment).

In case of macro nutrient content, Table 7 and 8 showed $\mathrm{N}, \mathrm{P}$ and $\mathrm{K}$ contents were higher with the treatment eight as same trend followed with micronutrient contents in leaves. Table 4 revealed second harvested (August) samples were higher accumulation of $\mathrm{N}(0.74$ $\mathrm{mg} / \mathrm{kg}), \mathrm{P}(0.40 \mathrm{mg} / \mathrm{kg})$ and $\mathrm{K}(0.68 \mathrm{mg} / \mathrm{kg})$ than other two harvested samples in 2009.

The amounts of the same were significantly lower with the fourth, fifth and sixth harvested samples in the year 2010 (Table 5) but treatment wise higher accumulation of N, P and $\mathrm{K}$ was observed where three biofertilizers were applied in combinations.

Further linear correlation matrices of macro and micro elements were analyzed for the year 2009 and 2010 with the ash content and revealed positive significant correlation with the ash content, being higher with the harvested samples in year 2009 than the year 2010, significant at $1 \%$ (Table 6 ). 
Table 4. Elemental analysis (Macro) in dried Stevia leaf in 2009 (Mean $\pm \mathrm{SEM}, \mathrm{n}=3$, from three replicated plots).

\begin{tabular}{|c|c|c|c|c|c|c|c|c|c|}
\hline \multirow[t]{2}{*}{ Ts } & \multicolumn{3}{|c|}{$\begin{array}{l}\text { First harvest in May } \\
\left(\mathrm{mg} \mathrm{kg}^{-1}, \text { dry wt basis) }\right.\end{array}$} & \multicolumn{3}{|c|}{$\begin{array}{c}\text { Second harvest in } \\
\text { August } \\
\left(\mathrm{mg} \mathrm{kg}^{-1}, \text { dry wt basis) }\right.\end{array}$} & \multicolumn{3}{|c|}{$\begin{array}{c}\text { Third harvest in } \\
\text { November } \\
\text { (mg kg }{ }^{-1} \text {, dry wt basis) }\end{array}$} \\
\hline & $\mathrm{N}$ & $\mathrm{P}$ & $\mathrm{K}$ & $\mathrm{N}$ & $\mathrm{P}$ & $\mathrm{K}$ & $\mathrm{N}$ & $\mathrm{P}$ & $\mathrm{K}$ \\
\hline $\mathrm{T}_{1}$ & $\begin{array}{c}0.42 \pm \\
0.002\end{array}$ & $\begin{array}{c}0.10 \pm \\
0.002\end{array}$ & $\begin{array}{c}0.50 \pm \\
0.002\end{array}$ & $\begin{array}{c}0.44 \pm \\
0.012\end{array}$ & $\begin{array}{c}0.12 \pm \\
0.004\end{array}$ & $\begin{array}{c}0.52 \pm \\
0.006\end{array}$ & $\begin{array}{c}0.42 \pm \\
0.004\end{array}$ & $\begin{array}{c}0.10 \pm \\
0.012\end{array}$ & $\begin{array}{c}0.50 \pm \\
0.004\end{array}$ \\
\hline $\mathrm{T}_{2}$ & $\begin{array}{c}0.46 \pm \\
0.004^{* *}\end{array}$ & $\begin{array}{l}0.12 \pm \\
0.004^{*}\end{array}$ & $\begin{array}{l}0.52 \pm \\
0.003^{*}\end{array}$ & $\begin{array}{c}0.50 \pm \\
0.014^{* * *}\end{array}$ & $\begin{array}{c}0.16 \pm \\
0.012^{* *}\end{array}$ & $\begin{array}{l}0.54 \pm \\
0.004^{* *}\end{array}$ & $\begin{array}{c}0.48 \pm \\
0.004^{* * *}\end{array}$ & $\begin{array}{c}0.14 \pm \\
0.006^{* * *}\end{array}$ & $\begin{array}{c}0.52 \pm \\
0.012^{* *}\end{array}$ \\
\hline $\mathrm{T}_{3}$ & $\begin{array}{c}0.58 \pm \\
0.012^{* * *}\end{array}$ & $\begin{array}{c}0.15 \pm \\
0.005^{* *}\end{array}$ & $\begin{array}{c}0.57 \pm \\
0.014^{* * *}\end{array}$ & $\begin{array}{c}0.62 \pm \\
0.002^{* * *}\end{array}$ & $\begin{array}{c}0.18 \pm \\
0.012^{* * *}\end{array}$ & $\begin{array}{c}0.58 \pm \\
0.012^{* * *}\end{array}$ & $\begin{array}{c}0.60 \pm \\
0.012^{* * *}\end{array}$ & $\begin{array}{c}0.15 \pm \\
0.004^{* * *}\end{array}$ & $\begin{array}{c}0.56 \pm \\
0.001^{* * *}\end{array}$ \\
\hline $\mathrm{T}_{4}$ & $\begin{array}{c}0.47 \pm \\
0.006^{* *}\end{array}$ & $\begin{array}{c}0.17 \pm \\
0.011^{* * *}\end{array}$ & $\begin{array}{c}0.59 \pm \\
0.011^{* * *}\end{array}$ & $\begin{array}{c}0.54{ }^{ \pm} \\
0.004^{* * *}\end{array}$ & $\begin{array}{c}0.19 \pm \\
0.014^{* * *}\end{array}$ & $\begin{array}{c}0.60 \pm \\
0.012^{* * *}\end{array}$ & $\begin{array}{c}0.533^{ \pm} \\
0.003^{* * *}\end{array}$ & $\begin{array}{c}0.16 \pm \\
0.012^{* * *}\end{array}$ & $\begin{array}{c}0.58{ }^{ \pm} \\
0.006^{* * *}\end{array}$ \\
\hline $\mathrm{T}_{5}$ & $\begin{array}{c}0.56 \pm \\
0.014^{* * *}\end{array}$ & $\begin{array}{c}0.22 \pm \\
0.012^{* * *}\end{array}$ & $\begin{array}{c}0.53 \pm \\
0.003^{* *}\end{array}$ & $\begin{array}{c}0.67 \pm \\
0.012^{* * *}\end{array}$ & $\begin{array}{c}0.28 \pm \\
0.016^{* * *}\end{array}$ & $\begin{array}{c}0.59 \pm \\
0.012^{* * *}\end{array}$ & $\begin{array}{c}0.64 \pm \\
0.013^{* * *}\end{array}$ & $\begin{array}{c}0.25 \pm \\
0.003^{* * *}\end{array}$ & $\begin{array}{c}0.57 \pm \\
0.003^{* * *}\end{array}$ \\
\hline $\mathrm{T}_{6}$ & $\begin{array}{c}0.58 \pm \\
0.012^{* * *}\end{array}$ & $\begin{array}{c}0.28 \pm \\
0.006^{* * *}\end{array}$ & $\begin{array}{c}0.56 \pm \\
0.006^{* *}\end{array}$ & $\begin{array}{c}0.70 \pm \\
0.013^{* * *}\end{array}$ & $\begin{array}{c}0.30 \pm \\
0.004^{* * * *}\end{array}$ & $\begin{array}{c}0.62 \pm \\
0.004^{* * *}\end{array}$ & $\begin{array}{c}0.688^{ \pm} \\
0.001^{* * *}\end{array}$ & $\begin{array}{c}0.28 \pm \\
0.002^{* * *}\end{array}$ & $\begin{array}{c}0.59 \pm \\
0.012^{* * *}\end{array}$ \\
\hline $\mathrm{T}_{7}$ & $\begin{array}{c}0.54 \pm \\
0.003^{* * *}\end{array}$ & $\begin{array}{c}0.31 \pm \\
0.004^{* * *}\end{array}$ & $\begin{array}{c}0.54 \pm \\
0.012^{* *}\end{array}$ & $\begin{array}{c}0.71 \pm \\
0.013^{* * *}\end{array}$ & $\begin{array}{c}0.37 \pm \\
0.002^{* * *}\end{array}$ & $\begin{array}{c}0.64 \pm \\
0.014^{* * *}\end{array}$ & $\begin{array}{c}0.69 \pm \\
0.014^{* * *}\end{array}$ & $\begin{array}{c}0.32 \pm \\
0.012^{* * *}\end{array}$ & $\begin{array}{c}0.60 \pm \\
0.004^{* * *}\end{array}$ \\
\hline $\mathrm{T}_{8}$ & $\begin{array}{c}0.59 \pm \\
0.003^{* * *}\end{array}$ & $\begin{array}{c}0.37 \pm \\
0.012^{* * *}\end{array}$ & $\begin{array}{c}0.60 \pm \\
0.003^{* * *}\end{array}$ & $\begin{array}{c}0.74 \pm \\
0.012^{* * *}\end{array}$ & $\begin{array}{c}0.40 \pm \\
0.002^{* * *}\end{array}$ & $\begin{array}{c}0.68 \pm \\
0.016^{* * *}\end{array}$ & $\begin{array}{c}0.71 \pm \\
0.006^{* * *}\end{array}$ & $\begin{array}{c}0.37 \pm \\
0.012^{* * *}\end{array}$ & $\begin{array}{c}0.63 \pm \\
0.014^{* * *}\end{array}$ \\
\hline
\end{tabular}

- $\quad$ Ts $=$ Treatments; $\mathrm{T}_{1}=$ Control $(\mathrm{FYM}), \mathrm{T}_{2}=\mathrm{FYM}+\mathrm{PSB}, \mathrm{T}_{3}=\mathrm{FYM}+\mathrm{AZO}, \mathrm{T}_{4}=\mathrm{FYM}+\mathrm{VAM}$,

$\mathrm{T}_{5}=\mathrm{FYM}+\mathrm{VAM}+\mathrm{AZO}$,

$\mathrm{T}_{6}=\mathrm{FYM}+\mathrm{PSB}+\mathrm{AZO}, \mathrm{T}_{7}=\mathrm{FYM}+\mathrm{PSB}+\mathrm{VAM}, \mathrm{T}_{8}=\mathrm{FYM}+\mathrm{PSB}+\mathrm{AZO}+\mathrm{VAM} ;$

- $\quad * \mathrm{P}<0.05, * * \mathrm{P}<0.01$ and $* * * \mathrm{P}<0.001 ; \mathrm{ns}=$ Non significant (compared with control treatment).

Table 5. Elemental analysis (Macro) in dried Stevia leaf in 2010 (Mean $\pm \mathrm{SEM}$, $\mathrm{n}=3$, from three replicated plots).

\begin{tabular}{|c|c|c|c|c|c|c|c|c|c|}
\hline \multirow[t]{2}{*}{ Ts } & \multicolumn{3}{|c|}{$\begin{array}{l}\text { First harvest in May } \\
\left(\mathrm{mg} \mathrm{kg}^{-1}, \text { dry wt basis) }\right.\end{array}$} & \multicolumn{3}{|c|}{$\begin{array}{c}\text { Second harvest in } \\
\text { August } \\
\left(\mathrm{mg} \mathrm{kg}^{-1}, \text { dry wt basis) }\right.\end{array}$} & \multicolumn{3}{|c|}{$\begin{array}{c}\text { Third harvest in } \\
\text { November } \\
\text { (mg kg }{ }^{-1}, \text { dry wt basis) }\end{array}$} \\
\hline & $\mathrm{N}$ & 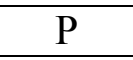 & $\mathrm{K}$ & $\mathrm{N}$ & $\mathrm{P}$ & $\mathrm{K}$ & $\mathrm{N}$ & $\mathrm{P}$ & $\mathrm{K}$ \\
\hline $\mathrm{T}_{1}$ & & & & & 0.012 & & & & \\
\hline $\mathrm{T}_{2}$ & $\begin{array}{c}0.45 \pm \\
0.006^{* * *}\end{array}$ & & & $\begin{array}{c}0.42 \pm \\
0.016^{* *}\end{array}$ & $\begin{array}{l}0.10 \pm \\
0.003^{\text {ns }}\end{array}$ & $\begin{array}{c}0.49 \pm \\
0.003^{* * *}\end{array}$ & $\begin{array}{c}0.40 \pm \\
0.016^{* * *}\end{array}$ & $\begin{array}{l}0.09 \pm \\
0.001^{*}\end{array}$ & $\begin{array}{c}0.45 \pm \\
0.012^{* * *}\end{array}$ \\
\hline $\mathrm{T}_{3}$ & $\begin{array}{c}0.59{ }^{ \pm} \\
0.001^{* * *}\end{array}$ & $\begin{array}{c}0.144^{ \pm} \\
0.003^{* * *}\end{array}$ & $\begin{array}{c}0.54^{ \pm} \\
0.012^{* * *}\end{array}$ & $\begin{array}{c}0.54 \pm \\
0.012^{* * *}\end{array}$ & $\begin{array}{l}0.12 \pm \\
0.014^{* *}\end{array}$ & $\begin{array}{c}0.51 \pm \\
0.002^{* * *}\end{array}$ & $\begin{array}{c}0.43 \underset{ }{ \pm} \\
0.012^{* * *}\end{array}$ & $\begin{array}{l}0.10 \pm \\
0.012^{* *}\end{array}$ & $\begin{array}{c}0.47 \pm \\
0.012^{* * *}\end{array}$ \\
\hline
\end{tabular}




\begin{tabular}{|c|c|c|c|c|c|c|c|c|c|}
\hline $\mathrm{T}_{4}$ & $\begin{array}{c}0.54 \pm \\
0.002^{* * *}\end{array}$ & $\begin{array}{c}0.15 \pm \\
0.002^{* * *}\end{array}$ & $\begin{array}{c}0.56 \pm \\
0.001^{* * *}\end{array}$ & $\begin{array}{c}0.50 \pm \\
0.012^{* * *}\end{array}$ & $\begin{array}{c}0.13 \pm \\
0.012^{* * *}\end{array}$ & $\begin{array}{c}0.52 \pm \\
0.004^{* * *}\end{array}$ & $\begin{array}{c}0.46 \pm \\
0.012^{* * *}\end{array}$ & $\begin{array}{c}0.11 \pm \\
0.006^{* * *}\end{array}$ & $\begin{array}{c}0.48 \pm \\
0.006^{* * *}\end{array}$ \\
\hline $\mathrm{T}_{5}$ & $\begin{array}{c}0.63 \pm \\
0.012^{* * *}\end{array}$ & $\begin{array}{c}0.23 \pm \\
0.002^{* * *}\end{array}$ & $\begin{array}{c}0.54 \pm \\
0.001^{* * *}\end{array}$ & $\begin{array}{c}0.57 \pm \\
0.001^{* * *}\end{array}$ & $\begin{array}{c}0.19 \pm \\
0.012^{* * *}\end{array}$ & $\begin{array}{c}0.52 \pm \\
0.006^{* * *}\end{array}$ & $\begin{array}{c}0.47 \pm \\
0.001^{* * *}\end{array}$ & $\begin{array}{c}0.14 \pm \\
0.003^{* * *}\end{array}$ & $\begin{array}{c}0.49 \pm \\
0.014^{* * *}\end{array}$ \\
\hline $\mathrm{T}_{6}$ & $\begin{array}{c}0.62 \pm \\
0.003^{* * *}\end{array}$ & $\begin{array}{c}0.27 \pm \\
0.016^{* * *}\end{array}$ & $\begin{array}{c}0.58 \pm \\
0.001^{* * *}\end{array}$ & $\begin{array}{c}0.60 \pm \\
0.004^{* * *}\end{array}$ & $\begin{array}{c}0.25 \pm \\
0.006^{* * *}\end{array}$ & $\begin{array}{c}0.56 \pm \\
0.012^{* * *}\end{array}$ & $\begin{array}{c}0.49 \pm \\
0.004^{* * *}\end{array}$ & $\begin{array}{c}0.18 \pm \\
0.012^{* * *}\end{array}$ & $\begin{array}{c}0.51 \pm \\
0.013^{* * *}\end{array}$ \\
\hline $\mathrm{T}_{7}$ & $\begin{array}{c}0.67 \pm \\
0.004^{* * *}\end{array}$ & $\begin{array}{c}0.30 \pm \\
0.014^{* * *}\end{array}$ & $\begin{array}{c}0.58 \pm \\
0.002^{* * *}\end{array}$ & $\begin{array}{c}0.62 \pm \\
0.002^{* * *}\end{array}$ & $\begin{array}{c}0.28 \pm \\
0.001^{* * *}\end{array}$ & $\begin{array}{c}0.57 \pm \\
0.003^{* * *}\end{array}$ & $\begin{array}{c}0.50 \pm \\
0.002^{* * *}\end{array}$ & $\begin{array}{c}0.20 \pm \\
0.006^{* * *}\end{array}$ & $\begin{array}{c}0.52 \pm \\
0.002^{* * *}\end{array}$ \\
\hline $\mathrm{T}_{8}$ & $\begin{array}{c}0.70 \pm \\
0.004^{* * *}\end{array}$ & $\begin{array}{c}0.35 \pm \\
0.002^{* * *}\end{array}$ & $\begin{array}{c}0.60 \pm \\
0.004^{* * *}\end{array}$ & $\begin{array}{c}0.64 \pm \\
0.002^{* * *}\end{array}$ & $\begin{array}{c}0.30 \pm \\
0.001^{* * *}\end{array}$ & $\begin{array}{c}0.58 \pm \\
0.012^{* * *}\end{array}$ & $\begin{array}{c}0.51 \pm \\
0.002^{* * *}\end{array}$ & $\begin{array}{c}0.24 \pm \\
0.012^{* * *}\end{array}$ & $\begin{array}{c}0.54 \pm \\
0.006^{* * *}\end{array}$ \\
\hline
\end{tabular}

- $\quad$ Ts $=$ Treatments; $\mathrm{T}_{1}=$ Control $(\mathrm{FYM}), \mathrm{T}_{2}=\mathrm{FYM}+\mathrm{PSB}, \mathrm{T}_{3}=\mathrm{FYM}+\mathrm{AZO}, \mathrm{T}_{4}=\mathrm{FYM}+\mathrm{VAM}$, $\mathrm{T}_{5}=\mathrm{FYM}+\mathrm{VAM}+\mathrm{AZO}$

$\mathrm{T}_{6}=\mathrm{FYM}+\mathrm{PSB}+\mathrm{AZO}, \mathrm{T}_{7}=\mathrm{FYM}+\mathrm{PSB}+\mathrm{VAM}, \mathrm{T}_{8}=\mathrm{FYM}+\mathrm{PSB}+\mathrm{AZO}+\mathrm{VAM} ;$

- $\quad * \mathrm{P}<0.05, * * \mathrm{P}<0.01$ and $* * * \mathrm{P}<0.001 ; \mathrm{ns}=$ Non significant (compared with control treatment).

Table 6. Correlation matrix between mean $\%$ Ash content and other elements in the year 2009 and 2010

\begin{tabular}{|c|c|c|c|c|c|c|c|c|}
\hline \multirow{2}{*}{} & \multicolumn{2}{|c|}{ Year 2009} & \\
\cline { 2 - 9 } & $\begin{array}{c}\text { Ash } \\
\text { content }\end{array}$ & $\mathrm{Fe}$ & $\mathrm{Mn}$ & $\mathrm{Cu}$ & $\mathrm{Zn}$ & $\mathrm{N}$ & $\mathrm{P}$ & $\mathrm{K}$ \\
\hline $\begin{array}{c}\text { Ash } \\
\text { content }\end{array}$ & 1.00 & & & & & & & \\
\hline $\mathrm{Fe}$ & $0.91^{* *}$ & 1.00 & & & & & & \\
\hline $\mathrm{Mn}$ & $0.87^{* *}$ & $0.98^{* *}$ & 1.00 & & & & & \\
\hline $\mathrm{Cu}$ & $0.93^{* *}$ & $0.95^{* *}$ & $0.94^{* *}$ & 1.00 & & & \\
\hline $\mathrm{Zn}$ & $0.87^{* *}$ & $0.97^{* *}$ & $0.99^{* *}$ & $0.95^{* *}$ & 1.00 & & & \\
\hline $\mathrm{N}$ & $0.88^{* *}$ & $0.87^{* *}$ & $0.88^{* *}$ & $0.95^{* *}$ & $0.89 * *$ & 1.00 & & \\
\hline $\mathrm{P}$ & $0.86^{* *}$ & $0.90^{* *}$ & $0.94^{* *}$ & $0.92^{* *}$ & $0.97 * *$ & $0.90^{* *}$ & 1.00 & \\
\hline $\mathrm{K}$ & $0.94^{* *}$ & $0.88^{*}$ & $0.88^{* *}$ & $0.84^{*}$ & $0.88^{* *}$ & $0.81^{*}$ & $0.84^{*}$ & 1.00 \\
\hline
\end{tabular}




\begin{tabular}{|c|c|c|c|c|c|c|c|c|}
\hline & \multicolumn{8}{|c|}{ Year 2010} \\
\hline & $\begin{array}{l}\text { Ash } \\
\text { content }\end{array}$ & $\mathrm{Fe}$ & Mn & $\mathrm{Cu}$ & $\mathrm{Zn}$ & $\mathrm{N}$ & $\mathrm{P}$ & $\mathrm{K}$ \\
\hline $\begin{array}{c}\text { Ash } \\
\text { content }\end{array}$ & 1.00 & & & & & & & \\
\hline $\mathrm{Fe}$ & $0.89 * *$ & 1.00 & & & & & & \\
\hline $\mathrm{Mn}$ & $0.84 *$ & $0.96 * *$ & 1.00 & & & & & \\
\hline $\mathrm{Cu}$ & $0.96 * *$ & $0.96 * *$ & $0.91 * *$ & 1.00 & & & & \\
\hline $\mathrm{Zn}$ & $0.82 *$ & $0.86^{* *}$ & $0.98 * *$ & $0.88 * *$ & 1.00 & & & \\
\hline $\mathrm{N}$ & $0.94 * *$ & $0.85^{*}$ & $0.86^{* *}$ & $0.93 * *$ & $0.81 *$ & 1.00 & & \\
\hline $\mathrm{P}$ & $0.83 *$ & $0.83 *$ & $0.92 * *$ & $0.87 * *$ & $0.95 * *$ & $0.92 * *$ & 1.00 & \\
\hline $\mathrm{K}$ & $0.95^{* *}$ & $0.89 * *$ & $0.89 * *$ & $0.96^{* *}$ & $0.88 * *$ & $0.97 * *$ & $0.92 * *$ & 1.00 \\
\hline
\end{tabular}

Significant at $1 \% ;{ }^{*}$ Significant at $5 \%$.

Table 7. Pooled biomass and respective total phenolic content in SR leaf in 2009 and 2010.

\begin{tabular}{|c|c|c|c|c|}
\hline \multirow{2}{*}{$\mathrm{T}$} & \multicolumn{2}{|c|}{ Year 2009 } & \multicolumn{2}{c|}{ Year 2010 } \\
\cline { 2 - 5 } & $\begin{array}{c}\text { Total pooled dried } \\
\text { biomass }(\mathrm{g})\end{array}$ & $\begin{array}{c}\text { Total phenolic content } \\
\text { (mg of Gallic acid per g of } \\
\text { extract) }\end{array}$ & $\begin{array}{c}\text { Total pooled dried } \\
\text { biomass }(\mathrm{g})\end{array}$ & $\begin{array}{c}\text { Total phenolic content } \\
\text { (mg of Gallic acid per g of } \\
\text { extract) }\end{array}$ \\
\hline $\mathrm{T}_{1}$ & $102.0 \pm 2.082$ & $40.76 \pm 0.023$ & $87 \pm 1.203$ & $36.55 \pm 0.017$ \\
\hline $\mathrm{T}_{2}$ & $154.3 \pm 1.155^{* * *}$ & $44.56 \pm 0.011^{* * *}$ & $94 \pm 1.320^{* * *}$ & $39.78 \pm 0.011^{\text {*** }}$ \\
\hline $\mathrm{T}_{3}$ & $168.3 \pm 1.202^{* * *}$ & $46.81 \pm 0.014^{* * *}$ & $104 \pm 2.031^{* * *}$ & $41.03 \pm 0.011^{* * *}$ \\
\hline $\mathrm{T}_{4}$ & $187.7 \pm 2.903^{* * *}$ & $52.96 \pm 0.011^{* * *}$ & $112 \pm 1.420^{* * *}$ & $43.80 \pm 0.011^{* * *}$ \\
\hline $\mathrm{T}_{5}$ & $253.3 \pm 2.208^{* * *}$ & $53.53 \pm 0.012^{* * *}$ & $123 \pm 1.202^{* * *}$ & $48.42 \pm 0.012^{* * *}$ \\
\hline $\mathrm{T}_{6}$ & $257.2 \pm 1.092^{* * *}$ & $56.85 \pm 0.017^{* * *}$ & $125 \pm 2.104^{* * *}$ & $48.95 \pm 0.017^{* * *}$ \\
\hline $\mathrm{T}_{7}$ & $262.9 \pm 1.023^{* * *}$ & $57.07 \pm 0.017^{* * *}$ & $127 \pm 1.089^{* * *}$ & $49.94 \pm 0.014^{* * *}$ \\
\hline $\mathrm{T}_{8}$ & $271.4 \pm 1.003^{* * *}$ & $64.52 \pm 0.014^{* * *}$ & $140 \pm 2.110^{* * * *}$ & $50.12 \pm 0.011^{* * *}$ \\
\hline
\end{tabular}

- $\mathrm{Ts}=$ Treatments; $\mathrm{T}_{1}=$ Control $(\mathrm{FYM}), \mathrm{T}_{2}=\mathrm{FYM}+\mathrm{PSB}, \mathrm{T}_{3}=\mathrm{FYM}+\mathrm{AZO}, \mathrm{T}_{4}=\mathrm{FYM}+\mathrm{VAM}$,

$\mathrm{T}_{5}=\mathrm{FYM}+\mathrm{VAM}+\mathrm{AZO}$,

$\mathrm{T}_{6}=\mathrm{FYM}+\mathrm{PSB}+\mathrm{AZO}, \mathrm{T}_{7}=\mathrm{FYM}+\mathrm{PSB}+\mathrm{VAM}, \mathrm{T}_{8}=\mathrm{FYM}+\mathrm{PSB}+\mathrm{AZO}+\mathrm{VAM} ;$

- $* \mathrm{P}<0.05, * * \mathrm{P}<0.01$ and $* * * \mathrm{P}<0.001 ; \mathrm{ns}=$ Non significant (compared with control treatment). 
- Correlation matrix between dried biomass and phenolic content is $r=0.96$; in year 2009 .

- Correlation matrix between dried biomass and phenolic content is $r=0.94$; in year 2010 .

- Correlation matrix of phenolic content is $r=0.92$, between year 2009 and 2010 .

\section{3. Determination of total phenolic content}

Results of the present study revealed that the level of the phenolic compounds in pooled dried leaves of SR (treatment wise) were found to be $64.52 \mathrm{mg} / \mathrm{g}$ and $50.12 \mathrm{mg} / \mathrm{g}$ which were higher with the treatment eight where three combinations of biofertilizers were applied in the year 2009 and 2010 respectively (Table 7). From the harvested samples in the year 2009, the results indicate that SR leaves contained very high amount of phenolic compound which was significant positively correlated with the increased dried biomass yield $(r=0.96)$ than harvested samples in year $2010(\mathrm{r}=0.94)$ and also was higher than that of control sample but lesser than that of standard gallic acid $(87.92 \mathrm{mg} / \mathrm{g})$. Table 7 also revealed the linear correlation ( $r$ ) of phenolic content between two years harvested sample was 0.92 .

\section{DISCUSSION}

\section{1. Effect of biofertilizers on physicochemical assessment}

Different physicochemical parameters like \% protein content, moisture content, ash value, SI and WAC were determined from all harvested Stevia crop, cultivated in acid soil zone of South India in the year 2009 and 2010. The results revealed second moth of harvested sample showed higher accumulation of \% protein, SI and WAC than the other harvested samples from both the years. Of late, protein is a basic component of metabolic regulation in plants which provides the path of varying enzymatic complement during the response to the environmental conditions (Huffakar and Peterson, 1974). One of the environmental conditions is application of biofertilizers. Biofertilizers are used to increase the yield of the biomass by uptaking the nutrients and accumulation in the leaf.

The increased effect was due to accumulation of the nutrients supplied by biofertilizers in the soil and hence increased the protein content in the leaf of SR during second time harvested samples in the year 2009, and the same content was decreased due to dilution effect of the nutrients in the plant. The result also confirmed the findings of Rakshit et al. (2002), who reported that the interaction between rhizobia and VAM fungi has received considerable attention because of the relatively high phosphorus demand for nitrogen fixation. It was reported that second harvest of plant resulted higher protein content than first harvested samples (Cupic et al., 2001). Further the effect of biofertilizers on Stevia plant cultivation and their effects on biomass yield were reported higher during pilot study in various soil environments (Das et al., 2009). It was also reported that biofertilizers had a favorable effect on growth and chemical composition of Jatropha curcus L. seedlings when studied in two different seasons (El-Quesni et al., 2013) and even it was reported that protein content increased after 45 days while at 30 and 60 days the content was least and in the middle phase of life cycle of plant protein content was highest (Patil, 2010) with applied biofertilizers.

The higher percent protein content of green pods of Pan 311 variety of cowpea in 2005/06 was due to its early maturity status and better nutrients uptake ability including nitrogen (Sebetha et al., 2010). The earlier studies were reported that \% protein in SR about $10 \%$ (Mishra et al., 2010) and even $20 \%$ (Tadhani and subhash, 2006). The present study was also followed the same trend and improved the protein content upto $21.29 \%$ due to 
maturity status and better nutrients uptake ability especially nitrogen with applied biofertilizers in combinations. The present study also showed that SI and WAC was dependent on protein content in the leaf of the plant. The content of SI increased with the increased protein content (Figure 2). It was reported that protein increased water holding capacity, when their swelling ability is enhanced (Mishra et al., 2010). Water absorption capacity of Stevia was recorded higher (Figure 3) with the second harvested samples in the year 2009 which might be due to higher protein content in SR leaf. Earlier study was revealed that WAC and SI in dried SR leaf were $4.7 \mathrm{ml} / \mathrm{g}$ and $5.01 \%$ respectively (Savita et al., 2004) whereas the results of the present investigation increased content of the same to the level of $5.12 \mathrm{ml} / \mathrm{g}$ and $6.42 \%$ which might be explained by higher amount of nitrogen accumulation in SR leaf with combined application of biofertilizers (Figure 4 \& 5).

Fig. 4. Effect of biofertilizers on \% protein content and swelling index (SI) in Stevia leaf (dry wt basis) in 2009 and 2010.

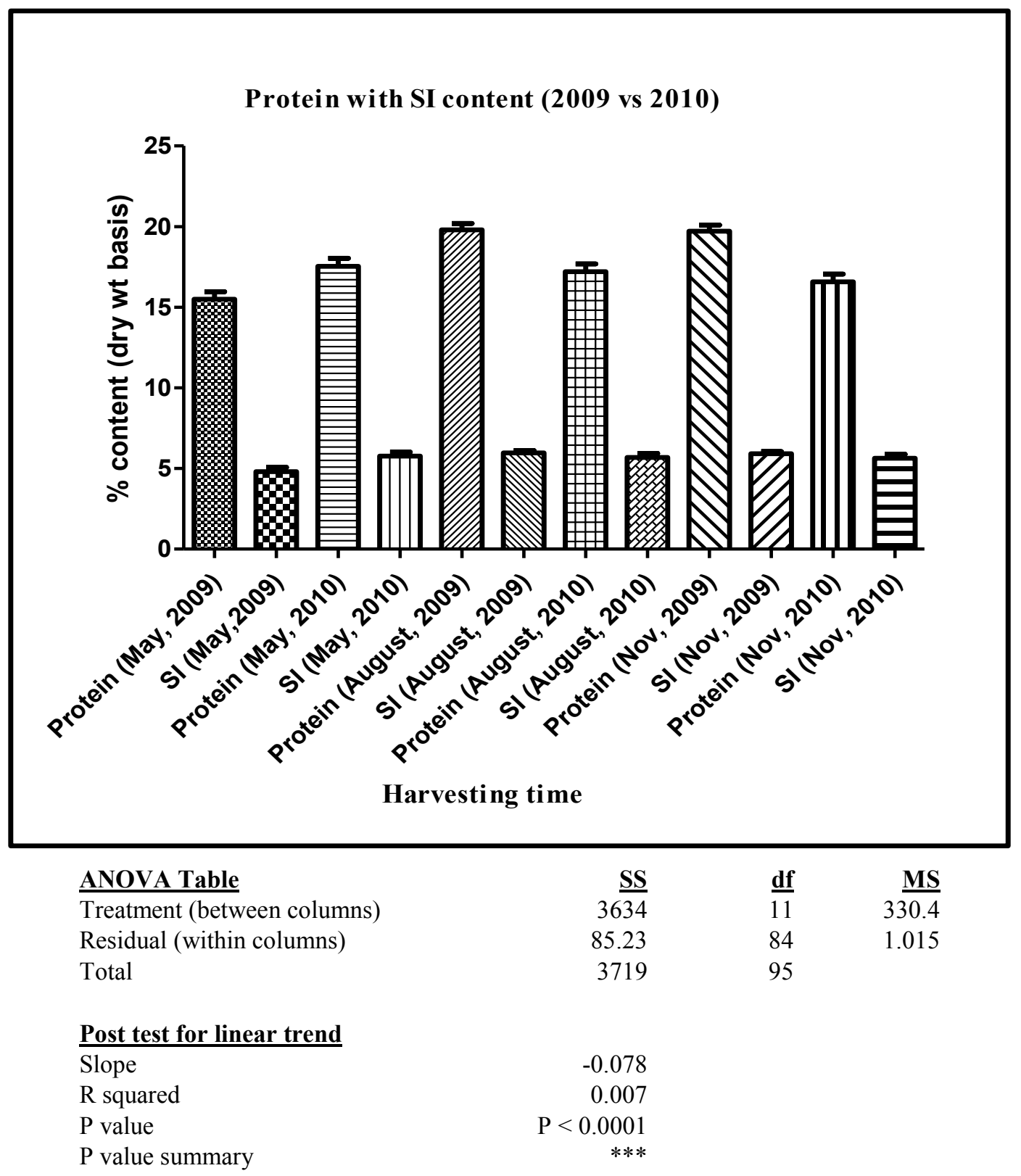


Fig. 5. Effect of biofertilizers on \% swelling index (SI) and water absorption capacity (WAC) in Stevia leaf (dry wt basis) in 2009 and 2010.

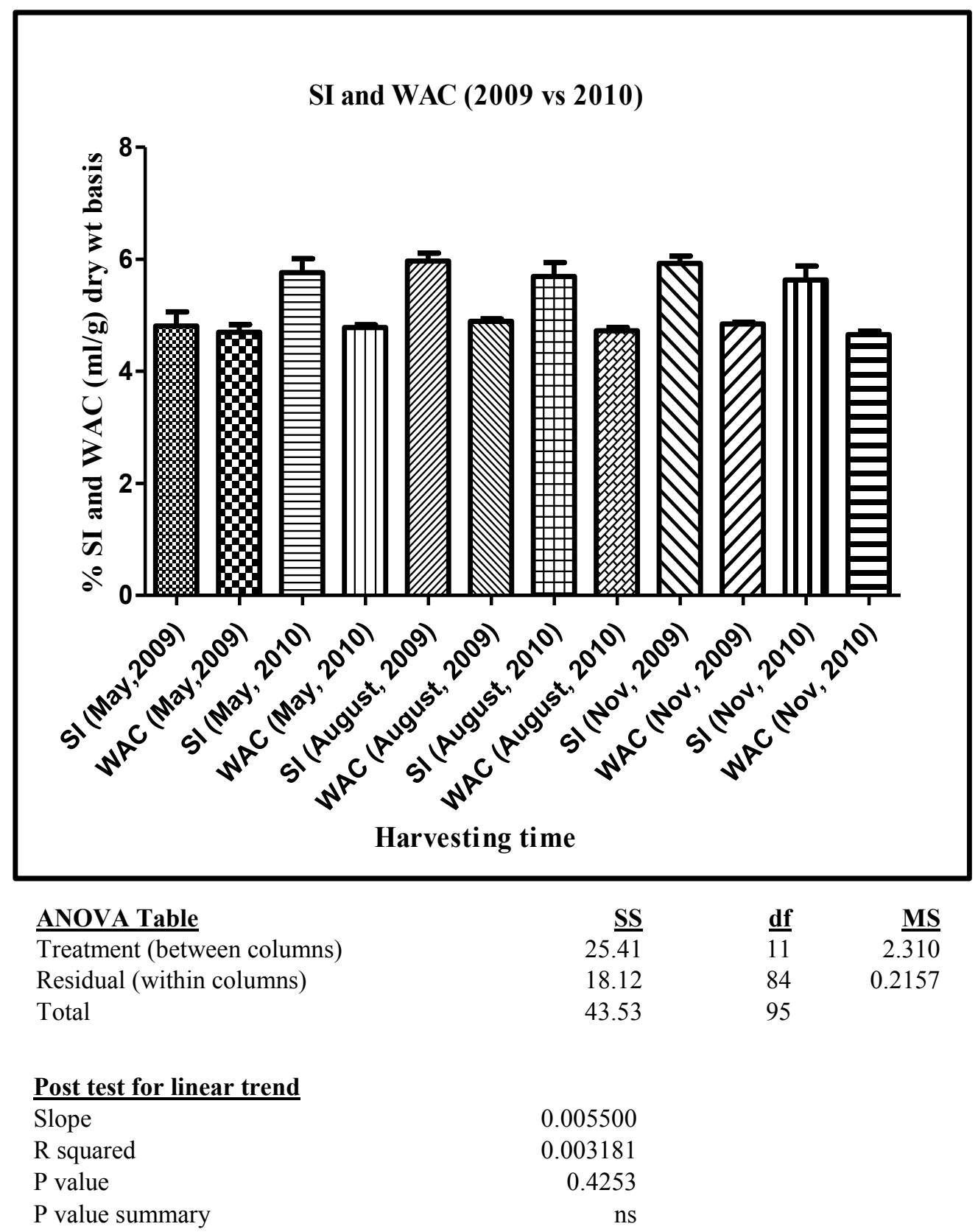

Interestingly, ash value content was higher up to third harvested samples than the other harvested samples, whereas moisture content was varied with the treatments in all the harvested samples of both the years. The ash content was higher than that of other treatments including control treatment during third harvest of the SR sample in the year 2009. The same trend was followed with the micro elements accumulation in SR leaf (Table 2). It was reported that ash content was dependent on the elemental content in the leaf (Dhiman et al., 2011 ) and its content were reported as $6.3 \%$ (Goyal et al., 2010), $7.41 \%$ (Abou-Arab et al., 
2010), 13.1\% (Tadhani and Subhash, 2006) whereas increased amount of ash content was investigated in the recent study with $13.54 \%$. Such increased ash content may be due to residual effect of the biofertilizers and also slow release of nutrients from fixed or adsorbed pools to the plant available pools into soil resulting more accumulation of micro elements in the leaf.

\section{2. Effect of biofertilizers on elemental analysis}

The content of microelements was greatly influenced by the applied biofertilizers. There were significant differences $(p<0.05)$ in the micronutrient composition of the SR leaf when compared with the control treatment and followed the same trend for all the harvested samples during both the years. The present study was recorded higher accumulation of Fe, $\mathrm{Mn}, \mathrm{Cu}$ and $\mathrm{Zn}$ in the leaf where combinations of three biofertilizers were applied simultaneously and being highest with third harvested sample. Such results were due to residual effect of the nutrients in the soil. The micronutrient content in the leaf of SR showed a high concentration of Fe (Table 2). Generally, iron is an essential element for synthesis of hemoglobin. Higher amount of iron in Stevia leaves accumulation is an additional benefit and importance for the maintenance of normal hemoglobin level in the body and hence, SR leaf can be used in various food preparations. A study was also reported that zinc and manganese are considered as antioxidant micro nutrients and their presence in leaf is essential to boost up the immune system (Jimoh and Oladiji, 2005) and in prevention of free radical mediated diseases. In case of $\mathrm{Cu}$ content in leaf, the same was also higher in the third harvested samples which showed an antagonistic action with protein content. It was reported that total protein content in sunflower seedlings decreased with increasing of $\mathrm{Cu}$ concentrations compared to the control seedlings, respectively (Zengin and Kirbag, 2007) which also confirmed the results of the present study where $\mathrm{Cu}$ concentration increased and decreased content of protein $(20.88 \%)$ with third harvested sample in year 2009 with the treatment $\mathrm{T}_{8}$ where three bio fertilizers were applied togetherly.

The content of macro elements (, P and $\mathrm{K}$ ) were significantly influenced by applied biofertilizers. The results revealed the higher accumulation of macronutrients in leaf of SR was in the second harvested samples during the year 2009 (Table 4) where combination of three biofertilizers was applied simultaneously The results of the present study are in agreement with findings of Anjanappa et al., 2011.

\section{3. Effect of biofertilizers and elemental accumulation in dried biomass on total phenolic content}

The total phenolic content in leaf of SR was significantly increased with the increased dried biomass as per treatment and was depicted in Table 7. It was seen that higher phenolic content was also followed the same trend with the treatment eight where three biofertilizers were applied in combination with respective higher pooled dried biomass. Overall results revealed that the harvested biomass content was higher during the year 2009 than harvested in 2010. SR already has its antioxidant nature as per earlier literatures (Tadhani et al., 2007; Shukla et al., 2009). Phenolic compounds have been shown to be responsible for the antioxidant activity of plant extracts (Rice-Evans et al., 1996; Gao et al., 2000). Phenols are one of the most important plant constituents because of their scavenging ability due to their hydroxyl groups (Hatano et al., 1989) and its contribute directly to antioxidant action (Duh et al., 1999). The present study showed the positive response as the earlier literature described by Javed and Panwar, (2013). Osuagwu and Edeoga (2012) were reported the effect of 
inorganic fertilizers (N, P and $\mathrm{K}$ ) on total phenolic content in Ocimum gratissimum leaves in dose dependent manner. The same trend followed in the present study with applied three biofertilizers resulted higher phenolic content over control sample.

\section{CONCLUSION}

The results may be concluded that second harvest during first year of the cultivation significantly increases $(\mathrm{p}<0.05)$ the percentage protein and macro nutrient contents $(\mathrm{N}, \mathrm{P}$ and $\mathrm{K}$ ) with simultaneous increase in the SI and WAC properties of the SR plant. Content of ash in dried SR leaf was also increased due to high accumulation of micro elements (Fe, Mn and $\mathrm{Zn}$ ) with the nutrients supplied by the biofertilizers. The results further reveal that with an increased content of physiochemical parameters, macro and micro elements in the dried leaf of SR plant the total phenolic contents in the SR plant also increased. The overall results suggest that Stevia can be used as an accessible source of natural antioxidants with consequent health benefits, preparation of various food products like different drinks, beverages and bakery products under balance use of bio-fertilizers.

\section{Acknowledgements}

Author was grateful to Mr Javali, Shimoga Karnataka, India, for providing Stevia plant cutting and cultivated land for the present investigation and Prof. Dr. Dilip Kumar Das, Former HOD, Dept. of Ag chemistry and Soil Science, B.C.K.V, Mohanpur, West Bengal, India for laboratory facilities to conduct protein and elemental analysis.

\section{Biography}

Kuntal Das, M.Pharm, Ph.D, is working as an assistant professor in Krupanidhi College of Pharmacy, Bangalore, India. He is having 09 years of experience in the field of Pharmacognosy. He has published more than 40 research papers, 3 books, 2 book chapters in many national and international journals. He is also serves as editor board member and reviewer for many reputed journals and delivered many oral presentations.

Prof. Dr. Raman Dang, is presently working as a Principal in Krupanidhi College of Pharmacy, Bangalore, India. $\mathrm{He}$ is having more than 20 years of experience in the field of Pharmacognosy. He has published more than 40 research papers, 2 book chapters in many national and international journals. He is also serves as editor board member and reviewer for many reputed journals and delivered many oral presentations. He is also served as an invited speaker from various countries.

\section{References}

[1] A. Abou-Arab, A. Abou-Arab, M. F. Abu-Salem, African Journal of Food Science 4 (2010) 269-281.

[2] M. Anjanappa, J. Venkatesha, B. Suresh Kumara, Vegetable Science 38 (2011) 58-62.

[3] AOAC, Official methods of Analysis of the Association of Official Analytical Chemists (15th edition), Washington DC. 992-995, 1990, USA.

[4] AOAC, Official Methods of Analysis. 16th ed. Association of Official Analytical Chemists, Washington, 780, 1995, USA. 
[5] AOAC, Association of Official Analytical Chemists. 17th Edn., Official Methods of Analysis of the Association of Official Analytical Chemists International 17th Ed. Published by the Association of Official Analytical Chemists International, Suite 400, 2200 Wilson Boulevard, Arlington, Virginia 22201-3301. 2000, USA.

[6] T. Cupic, S. Grljusic, S. Popovic, M. Stjepanovic, M. Tucak, Delgado I. (ed.), Lloveras J. (ed.). Quality in lucerne and medics for animal production. Zaragoza : CIHEAM. 45 (2001) 215-218. (Option s Méditerranéennes: Série A. Séminaires Méditerranéens.). Article available in http:/om.cih eam.org/article.ph p?ID PD F=1 600086.

[7] K. Das, R. Dang, T. N. Shivananda, Archives of Agronomy and Soil Science 55 (2009) 359-366

[8] A. Dhiman, A. Nanda, S. Ahmad, S, Toxicol Int. 18 (2011) 163-167.

[9] P. D. Duh, Y. Y. Tu, G. C. Yen, Lebensm.-Wiss. Technol 32 (1999) 269-277.

[10]F. E. M. El-Quesni, Kh. I. Hashish, M. M. Kandil, A. A. M. Mazher, World Applied Sciences Journal 21 (2013) 927-932.

[11]D. Fronza, M. V. Folegatti, Scientia Agricola 60 (2003) 595-599.

[12]X. Gao, M. Ohlander, N. Jeppsson, L. Bjork, V. Trajkovski, J. Agric. Food Chem 48 (2000) 1485-1490.

[13]S. Ghanta, A. Banerjee, A. Poddar, S. Chattopadhyay, Journal of Agricultural Food Chemistry 55 (2007) 10962-10967.

[14]S. Goyal, S. Samsher, R. Goyal, International Journal of Food Sciences and Nutrition 61 (2010) 1-10.

[15]T. Hatano, R. Edamatsu, A. Mori, Chem. Pharm. Bull 37 (1989) 2016-2021.

[16]P. C. H. Hollman, M. G. L. Hertog, M. B. Katan, Food Chem 57 (1996) 43-46.

[17]R. C. Huffaker, L. W. Peterson, Ann. Rev. P1. Physiol 25 (1974) 363-392.

[18]S. Javed, A. Panwar, Recent Research in Science and Technology 5 (2013) 40-44.

[19]F.O. Jimoh, A.T. Oladiji, Africani. Biotechnol 4 (2005) 1439-1442.

[20]D. Kim, S. Jeong, C. H. Lee, Food Chem 81 (2003) 321-326.

[21]N. W. Megeji, J. K. Kumar, V. Singh, V. K. Kaul, P. S. Ahuja, Current Sci. 88 (2005) 801-804.

[22]P. Mishra, R. Singh, U. Kumar, V. Prakash, Global Journal of Biotecnology \& Biochemistry 5 (2010) 62-74.

[23]D. Mowrey, Op. Cit (1992) 1-14.

[24]P. K. Mukherjee, Quality Control of Herbal Drugs, Business Horizons Pharmaceutical Publishers, G-59, Masjid Moth, GK-2, New Delhi - 110048, 2nd Reprint Edn, 167, 438448 (2007) 452.

[25]G. G. E. Osuagwu, H. O. Edeoga, Int. J. Med. Arom. Plant 2 (2012) 254-262.

[26]N. M. Patil, Recent Research in Science and Technology 2 (2010) 42-44. 
[27]I. Prakash, G. Dubois, J. Clos, K. Wilkens, L. Fosdick, Food and Chemical Toxicology 46 (2008) S75-S82.

[28]A. Rakshit, P. B. S. Bhadoria, D. K. Das, J Interacademica 6 (2002) 570-574.

[29]C. Rice-Evans, N. Miller, G. Paganga, Free Radical Biology and Medicine 20 (1996) 933-956.

[30] R. D. Rosario, D. M. Flores, J. Sci. Food Agric 32 (1981) 175-180.

[31]S. Savita, K. Sheela, S. Sunanda, A. Shankar, P. Ramakrishna, Journal of Human Ecology 15 (2004) 261-264.

[32]E. T. Sebetha, V. I. Ayodele, F. R. Kutu, I. K. Mariga, African Journal of Biotechnology 9 (2010) 628-634.

[33]S. Shukla, A. Mehta, V. K. Bajpai, S. Shukla, Food and Chemical Toxicology 47 (2009) 2338-2343.

[34]K. Slinkard, V. L. Singleton, Am. J. Enol. Viticult 28 (1977) 49-55.

[35]S. E. Smith, D. J. Read, Mycorrhizal Symbiosis, 2nd Edn. Academic Press, San Diego (1997) 126.

[36]D. Soejarto, A. Kinghorn (Ed.), Stevia: The genus Stevia. London, New York: Taylor and Francis (2002) 18-39.

[37]M. Tadhani, R. Subhash, Journal of Medical Sciences 6 (2006) 321-326.

[38] M. B. Tadhani, V. H. Patel, R. Subhash, Journal of Food Composition and Analysis 20 (2007) 323-329.

[39]F. K. Zengin, S. Kirbag, Journal of Environmental Biology 28 (2007) 561-566. 